Preprint typeset in JHEP style - HYPER VERSION

\title{
Brane Induced Gravity, its Ghost and the Cosmological Constant Problem
}

\author{
S. F. Hassan \\ Department of Physics 83 The Oskar Klein Centre for Cosmoparticle Physics, \\ Stockholm University, AlbaNova University Centre, SE-106 91 Stockholm, Sweden \\ E-mail: fawad@fysik.su.se

\section{Stefan Hofmann} \\ Arnold Sommerfeld Center for Theoretical Physics, LMU, \\ Theresienstr. 37, 80333 Munich, Germany, 83 \\ Excellence Cluster Universe, Boltzmannstr. 2, 85748 Garching, Germany \\ E-mail: stefan.hofmann@physik.lmu.de

\section{Mikael von Strauss \\ Department of Physics \& The Oskar Klein Centre for Cosmoparticle Physics, Stockholm University, AlbaNova University Centre, SE-106 91 Stockholm, Sweden \\ E-mail: mvs@fysik.su.se}

\begin{abstract}
Brane Induced Gravity" is regarded as a promising framework for addressing the cosmological constant problem, but it also suffers from a ghost instability for parameter values that make it phenomenologically viable. We carry out a detailed analysis of codimension $>2$ models employing gauge invariant variables in a flat background approximation. It is argued that using instead a curved background sourced by the brane would not resolve the ghost issue, unless a very specific condition is satisfied (if satisfiable at all). As for other properties of the model, from an explicit analysis of the 4-dimensional graviton propagator we extract a mass, a decay width and a momentum dependent modification of the gravitational coupling for the spin 2 mode. In the flat space approximation, the mass of the problematic spin 0 ghost is instrumental in filtering out a brane cosmological constant. The mass replaces a background curvature that would have had the same function. The optical theorem is used to demonstrate the suppression of graviton leakage into the uncompactified bulk. Then, we derive the 4-dimensional effective action for gravity and show that general covariance is spontaneously broken by the bulk-brane setup. This provides a natural realization of the gravitational Higgs mechanism. We also show that the addition of extrinsic curvature dependent terms has no bearing on linearized brane gravity.
\end{abstract}

KEYwords: Modified gravity, Cosmological applications of theories with extra dimensions. 


\section{Contents}

1. Introduction and Results 2

1.1 An overview of BIG, its promise and problems 2

1.2 Overview and discussion of our results

1.3 Organization of the paper

2. Preliminaries 8

2.1 The model and its origins 8

2.2 Flat background and gauge invariant variables

2.3 Thick branes and "blurred" quantities 10

2.4 Bulk-brane relations 11

3. The Basic Brane Induced Gravity Model 12

3.1 Equation of motion and solutions 12

3.2 Graviton decay from optical theorem 15

3.3 Graviton mass, decay width and coupling 16

4. Screening of $\Lambda$ and the Origin of Tachyon/Ghost Problem 20

4.1 Filtering out of brane cosmological constant 21

4.2 On the origin of the tachyon and ghost problems

4.3 Implication for couplings 24

4.4 Contrast with Fierz-Pauli Massive gravity 25

5. Inclusion of Extrinsic Curvature Terms 25

5.1 Extrinsic curvature term in the action 26

5.2 Equations of motion with extrinsic curvature contributions 26

5.3 Solutions with extrinsic curvature contributions 27

6. Revisiting Fierz-Pauli Massive Gravity 29

6.1 Fierz-Pauli massive gravity in gauge invariant variables 29

6.2 A 1-parameter family of actions for FP gravity 31

7. 4-d Effective Action in BIG and Broken Gauge Invariance 32

7.1 4-dimensional effective action in Brane Induced Gravity 32

7.2 A 1-parameter family of 4-d BIG effective actions 34

7.3 Spontaneous breaking of 4-d gauge invariance 36

8. Conclusions 37

A. Projection operators for gauge invariant variables 38 


\section{Introduction and Results}

"Brane induced gravity" (BIG) [1, 2] (for a review see [3]) is regarded as providing one of the few promising frameworks for addressing the cosmological constant problem [i] in the sense of explaining how the observed value of vacuum energy could be so small as compared to the types of values inferred from quantum field theory [5, 6] (other approaches include [7, 8], for reviews see [9, 10, 11]). In its basic form, the model regards the 4-dimensional universe as a 3 -brane in a non-compact $(4+n)$-dimensional bulk spacetime. Gravitational dynamics arise from Einstein-Hilbert actions both in the bulk and on the brane. Bulk gravity absorbs the effect of brane cosmological constant, leaving behind effectively 4dimensional gravity on the brane with a "filtered out" cosmological constant. Unfortunately consistent constructions are still lacking, for example, issues related to the presence of a tachyonic ghost mode have not been resolved satisfactorily. Hence more work is needed to obtain consistent working models of this type ${ }^{1}$.

\subsection{An overview of BIG, its promise and problems}

Let's briefly review the origin, workings and shortcomings of the model.

Historical background and motivation: In ordinary Einstein-Hilbert gravity a cosmological constant $\Lambda$ in a $3+1$ dimensional spacetime gives rise to a de Sitter metric. However, if this specetime is a 3 -brane embedded in a $4+n$ dimensional bulk, then it is known that bulk gravity has classical solutions in which $\Lambda$ (which is the same as the brane tension) curves directions transverse to the brane while the metric along the 3-brane remains flat, provided $n>1$ [14, 15, 16]. This eliminates $\Lambda$ from the brane point of view at the level of classical solutions. But in this model, gravity is a $(4+n)$-dimensional force.

To get effectively 4-dimensional gravity on the brane without compactifying the extra dimensions, Dvali, Gabadadze and Porrati [1, 2] proposed adding to the bulk gravity action, a 4-dimensional Einstein-Hilbert action for the brane induced metric. Such brane localized actions, along with extra corrections, naturally arise in field theory [17] as well as in string theory [18, 19, 20, 21, 22, 23] setups, hence the name brane induced gravity. However, for phenomenological reasons, the parameters have to be tuned to make the brane localized action dominant. The $n=1$ case is the well studied DGP model [1, 3]. It is special in that it does not suffer from tachyon/ghost problems. But it is also not adequate for addressing the cosmological constant problem in the sense stated above. In [5, 6] it was argued that models with $n>2$ should be able to filter out a brane cosmological constant, making gravity on the brane less sensitive to it, while at the same time giving rise to essentially 4 dimensional gravitational interactions on the brane. These arguments were mainly based on the classical solutions of 14, 15 described above ${ }^{2}$.

Beside the classical solutions, most of the explicit work on BIG with $n>2$ has focused, for reasons of technical simplicity, on the $\Lambda=0$ case, analyzing the propagation of

\footnotetext{
${ }^{1}$ In this paper we will not discuss variants of the basic setup like cascading gravity 12 that address the ghost issue 13 .

${ }^{2}$ The $n=2$ models also filter out $\Lambda$ but they share features of both $n=1$ and $n>2$ models and are discussed in some detail in $24,25,26$.
} 
linearized metric fluctuations for a flat brane, in flat bulk spacetime. The purpose is to, from this, glean information on infrared modifications of gravity that would eventually result in filtering out $\Lambda$. In particular, one finds that for appropriate values of parameters in the action, gravity can be made effectively 4-dimensional over a desired range of distances. It will also contain massive unstable gravitons [2, 5, 6, 27]. Beyond this range (say, for distances of the order of the size of the Universe), the emergent unstable graviton, with a small but momentum dependent mass, produces infrared modifications of gravity. This is supposed to be relevant to filtering out $\Lambda$, as suggested by the classical solutions mentioned above.

Problematic issues: The above picture looks promising, except for some important issues that arise in the analysis carried out so far, and that need to be better understood and resolved. We describe five issues below.

1. The evidence in [5, 6] that BIG would filter out a brane cosmological constant is mostly based on the existence of the classical solutions of [14, 15] that are supposed to describe how $\Lambda$ curves the space transverse to the brane. However a closer examination shows that in the vicinity of the brane, the source structure implied by these solutions does not correspond to the brane source (which is a common problem of brane solutions). This means that a detailed analysis of fluctuations (needed to obtain the effective 4-dimensional gravity on the brane) cannot be carried out around existing classical solutions ${ }^{3}$. To avoid this problem, in this paper we start with a flat background approximation and try to glean information about the effects of background curvature sourced by the brane.

2. Second, as pointed out in [28], the analysis of BIG in a flat background approximation indicates the presence of a tachyonic ghost mode and, therefore, an inconsistency of the model. This is a major drawback and resolving the ghost problem is crucial for developing these models further. It is easy to see that a ghost generically appears in massive gravity theories unless it is given an infinite mass, as in Fierz-Pauli gravity [29, 30, 31, 32, 33]. But in BIG around flat background, the tachyonic ghost cannot be avoided easily. There have been attempts to evade the problem by modifying the setup [13, 27, 34], but a satisfactory resolution is still lacking.

3. The third issue is the mode of realization of the gauge symmetry, in this case 4dimensional general covariance on the brane. This is related to the structure of the effective theory on the brane. It is known that in both Fierz-Pauli massive gravity, as well as in Higgs gravity setups (see [35, 36]), a graviton mass is related to the breaking of 4-d general covariance. However, in BIG, the starting theory is manifestly invariant under bulk and brane gauge transformations, while at the same time 4-d gravitons acquire an effective mass. In usual treatments this result is obtained after gauge fixing which obscures the nature of symmetries of the effective theory. How is the generation of mass related to the realization of symmetry in BIG models?

\footnotetext{
${ }^{3}$ Progress in this direction will be reported elsewhere.
} 
4. The fourth issue is a more detailed understanding of the technical aspects of the resulting modified gravity on the brane and its response to the cosmological constant, both in flat and curved background approximations. Technically, a zero thickness brane should consistently be replaced by one with an effective width [2, 17, 23], before a quantitative investigation of graviton mass, its decay and other possible modifications of the gravitational force. It is also important to see explicitly how a cosmological constant affects the situation. In this paper such an analysis is carried out in the flat background approximation.

5. Related to this is the fifth issue of naturalness of scales in BIG, at least based on some elementary considerations. The basic BIG model contains bulk and brane Planck scales, say, $M_{*}$ and $M_{P}$, respectively, as free parameters. For a realistic $M_{P} \sim 10^{19} \mathrm{GeV}$, to insure that the resulting IR modifications of gravity are not too large, the bulk gravity scale $M_{*}$ should be unnaturally low $\left(<10^{-3} \mathrm{eV}\right.$ for $\left.n \geq 2\right)$, corresponding to strongly coupled bulk gravity [2]. The effective brane thickness introduces another scale, though often this is taken to be related to $M_{*}$ [17, 23]. But a non-zero $\Lambda$ also affects the identification of the gravitational constant and other standard model couplings, influencing the problem of scales. Taking this into account is important since as a viable theory BIG should remain calculable at the interacting level and should not generate new hierarchy problems. It is not obvious that BIG could meet these requirements.

Considering the potential ramifications of brane induced gravity for the cosmological constant problem, we revisit these models and analyze them in some depth in the hope of gaining a better understanding of the issues outlined above. This work address some of these issues directly, and hopefully sheds light on, and sharpens the context of, the rest.

\subsection{Overview and discussion of our results}

This subsection contains a description of our results and their implications. We concentrate mainly on codimension $n>2$ BIG models relevant to the cosmological constant problem and allow for the addition of extrinsic curvature terms and a brane cosmological constant (brane tension) $\Lambda$. In this model, we study linearized metric fluctuations around a background configuration which is approximated as a flat 3-brane in a flat bulk spacetime (for the reason explained under item (1) of the previous subsection). The crucial point is that the presence of a small brane cosmological constant does not invalidate the flat background approximation.

Spontaneous breaking of general covariance: The basic variables are the bulk metric $G_{M N}$ and the brane embedding functions $x^{M}(\sigma)$. The symmetries are the brane and bulk general coordinate transformations, involving $\sigma^{\mu}$ and $X^{M}$, respectively. The standard gauge choice (Monge or static gauge for the brane, and harmonic gauge for the bulk symmetries) is natural for bulk physics but obscures the nature of symmetries of the effective brane theory. Thus, with a focus on 4-dimensional physics, we work with gauge invariant variables and the induced metric $g_{\mu \nu}=G_{M N} \partial_{\mu} x^{M} \partial_{\nu} x^{N}$, that naturally couples 
to brane matter. The outcome is that, beside the usual gauge invariant parts of the metric, we encounter 4 new gauge invariant variables. These are closely related to Stückelberg fields used to restore gauge invariance in Fierz-Pauli massive gravity. The 4-dimensional effective action, obtained after integrating out all extra dimensional modes, depends on these variables, and through them, on gauge dependent components of the metric. This explicitly demonstrates how 4-dimensional general covariance is spontaneously broken by the bulk-brane setup in the effective 4-dimensional theory, whereas all symmetries are manifest in the starting action. Thus, BIG naturally implement a gravitational Higgs mechanism of the sort discussed in [35, 36]. These issues are discussed in sections 6 and 7 .

Explicit analysis of graviton mass and decay: To study graviton propagation on the brane, one has to consider thick branes to avoid divergences associated with vanishing brane width. It is then known that integrating out bulk modes contributes a "selfenergy" term to the 4-dimensional graviton propagator, giving, schematically (see equations (3.15,3.16, 2.13) ),

$$
\frac{O_{\mu \nu \rho \sigma}^{(0,2)}}{B k^{2}+A \omega^{n-2} N^{(0,2)} \Sigma(k)} .
$$

$A$ and $B$ are inverse Newton constants in $d$ and 4 dimensions, $\omega$ is the brane width and the superscripts refer to spin 0 and 2 modes of graviton. The scale of graviton mass is set by $A \omega^{n-2} / B$, taken to be very small for phenomenological reasons.

1. After reviewing the well known problem of the tachyonic ghost nature of the spin 0 mode, we use a Gaussian profile for the brane thickness to explicitly extract from $\Sigma(k)$ a mass term, a decay width and a momentum dependent modification of the Newton constant for the healthy spin 2 mode. All this hinges on the fact that, as we show, the standard perturbative treatment of unstable particles in quantum field theory remains applicable for small graviton masses.

2. The suppression of the probability for graviton escape into the bulk, resulting in effectively 4-dimensional gravity on the brane, is demonstrated using the optical theorem.

Suppression of cosmological constant: In Einstein-Hilbert gravity, a cosmological constant $\Lambda$, however small, destabilizes flat space, giving rise to de Sitter or anti de Sitter spacetimes. In BIG, a brane $\Lambda$ can curve directions transverse to the brane, leaving the brane metric flat. However, in the analysis of BIG around a flat background, commonly employed to extract more detailed information about brane gravity, the setup does not allow for absorbing $\Lambda$ in the curvature of extra dimensions. But, as discussed in section 4 , the theory compensates for this and neutralizes $\Lambda$ using a $\Sigma(0) \neq 0$ piece of the self-energy in the scalar mode propagator, closely related to its tachyonic mass. This leaves the brane metric flat ${ }^{4}$, and only modifies gravitational and non-gravitational couplings. Based on this analysis, the following observations should be made:

\footnotetext{
${ }^{4}$ The $n>2$ BIG only attempts to explain why one does not observe a large cosmological constant. At least in this form, it does not explain the observed recent accelerated expansion due to a very small $\Lambda$.
} 
1. As far as filtering out $\Lambda$ is concerned, the tachyonic mass of the scalar ghost replaces the function of a background curvature (as an artifact of flat background approximation) and is not unhealthy. In fact, the mass is tachyonic only with respect to the wrong sign of the ghost kinetic term, but has a positive contribution to the energy.

2. The momentum dependence of $\Sigma(k)$, which determines the graviton decay width and normalizes the gravitational coupling, has no bearing on the suppression of $\Lambda$ (which is entirely due to $\Sigma(0)$ ). This is to be contrasted with the more general degravitation mechanisms [37] where a momentum dependent gravitational coupling could make the theory less sensitive to $\Lambda$. But the outcome of $n>2$ BIG is closer to the scenario in [38], more closely modeled around massive gravity.

3. In some of the literature on BIG, the suppression of $\Lambda$ is heuristically explained in terms of 3 -brane gravity becoming $4+n$-dimensional, hence weaker, at very large distances. This truly is the behaviour of $n=1$ theory which, however, does not filter out $\Lambda$. A closer inspection of the propagator in $n>2$ models shows that brane gravity at large distances remains 4 -dimensional and is dominated by $\Sigma(0)$, related to graviton mass, which suppresses $\Lambda$.

4. A lesson from this analysis is that the flat background approximation to BIG remains valid in the presence of a small cosmological constant, with implications for the resolution of the ghost problem to be discussed below.

Extrinsic curvature effects: Derivations of the BIG action in string theory setups also produce terms dependent on the extrinsic curvature of the brane. One may wonder if neglecting these terms has an effect on the ghost problem. The analysis in section 5 shows that these terms have no effect on brane gravity at the linearized level. This may be disappointing from the point of view of the ghost problem but also shows that these terms do not drastically modify gravity on the brane.

Status of the ghost problem: In equation (1.1) for the graviton propagators in BIG, $O_{\mu \nu \rho \sigma}^{(0,2)}$ contain the tensor structure. This is exactly the same as the tensor structure of Einstein-Hilbert gravity in 4 dimensions (obtainable by setting $A=0$ ). It is known that with this tensor structure, the theory is ghost free only for zero masses. Any non-zero mass pole implies a ghost, as is the case with $\mathrm{BIG}^{5}$. Resolving this problem is crucial for any eventual application of BIG. We do not achieve this in this paper, but sharpen the context of the problem.

1. As a warm up consider the following puzzle: The BIG action can arise within seemingly consistent setups, like the string theory setup of 18. How can these consistent setups lead to a ghost inconsistency? To answer this, note that the identification of ghost in BIG hinges on the $\Sigma$ term in (1.1) being a small self-energy correction. This

\footnotetext{
${ }^{5}$ The ghost can also be avoided by changing the tensor structure gravity in BIG for example, as in [27, 34] but that introduces vDVZ type discontinuity in the free theory. These could be avoided in the non-linear regime by the Vainshtein mechanism [39, 40], but we will not consider such alternatives here.
} 
is obviously the case in the phenomenologically interesting regime of small graviton mass $\left(\sim A \omega^{n-2} / B\right)$ which requires that the brane Einstein-Hilbert action dominates over the bulk one. If this is not the case, then either the ghost may have a large mass, beyond the regime of validity of the low-energy effective action, or $\Sigma(k)$ may contain appreciable corrections to $B k^{2}$ making the above interpretation of the propagator meaningless. This is the case in the string theory regime of [18] where, for a small string coupling $g_{s}, A \sim 1 / g_{s}^{2}$ dominates over $B \sim 1 / g_{s}$ (assuming $\omega \sim g_{s}^{0}$ ), the propagator is essentially $\Sigma^{-1}(k)$, and gravity is $d$-dimensional and ghost free. On the other hand, assuming $\omega^{4} \sim g_{s}$ and $n=4$ (more natural for D3-branes), the mass is at string scale.

2. Could the appearance of ghost (in the phenomenologically interesting cases) be related to the use of flat background approximation, ignoring $\Lambda$ and its back reaction on bulk geometry? As discussed in section 4, since our analysis is already valid for a small $\Lambda$, one can answer this in the negative by a continuity argument: that in any case, the small $\Lambda$ limit should correspond to our result which is not ghost free.

One may formulate a sharper criterion: In a gravitational background sourced by a brane $\Lambda$, the metric along the brane remains flat, hence the background mostly affects gravitational dynamics in the bulk. In terms of the propagators in (1.1), the curved background mostly affects the $A$-dependent self-energy term $\Sigma$, leaving $B k^{2}$ and tensor structure unchanged. Then in the phenomenologically interesting regime of small mass, where the $B$ term dominates, the propagator has the same structure as in flat background. The discussion above then shows that the ghost mode can be avoided only if the new self-energy term satisfies $\Sigma_{\text {curved }}(0)=0$, implying no hard masses. This can be regarded as the constraint on admissible ghost free backgrounds, if any. For more on this see the concluding section.

3. In the 4-dimensional effective action for BIG derived in section 7, the ghost can be related to the Stückelberg-like fields that arise in the gauge invariant formalism, and hence to the spontaneous breakdown of general covariance. This underlines the similarity with the ghost in standard massive gravity and in Higgs gravity.

\subsection{Organization of the paper}

The rest of the paper is organized as follows: In section 2, we introduce the model and detail the setup for the analysis. Gauge invariant variables are introduced and a brane thickness profile is used to avoid divergences associated with zero-thickness branes.

In section 3, we solve the equations for the basic BIG model and review the tachyonic ghost problem. The suppression of graviton escape into bulk is described in terms of the optical theorem. We then carry out an explicit analysis of the graviton propagator, extracting from it a mass, a decay width and a modulation of the gravitational coupling. It is shown in the process that standard perturbative QFT methods remain applicable for small graviton masses.

In section 4 , we consider the effect of a small brane cosmological constant $\Lambda$ in the flat background approximation, showing that for codimension $n>2$, it is filtered out by the 
tachyonic scalar mass. The tachyon problem is dismissed. The ghost problem is argued to survive, even when the theory is analyzed in a background sourced by $\Lambda$, except for special backgrounds (if they exist at all) that do not make brane gravity massive.

In section 5, we solve the equations in the presence of extrinsic curvature terms that generically arise in derivations of the BIG action. It is shown that they have no effect whatsoever on the brane gravity.

In section 6, we revisit massive Fierz-Pauli gravity with Stückelberg fields in terms of gauge invariant variables. In particular, it is shown that the correct solutions can be obtained from a 1-parameter family of actions.

In section 7 , we derive an effective action for the brane gravity by integrating out bulk related modes. We construct a 1-parameter family of effective actions that reproduce the correct solution and, generically, contain Stückelberg fields. This shows that gauge dependent modes of metric do not decouple, implying a spontaneous breakdown of 4dimensional general covariance. The relation to the gravitational Higgs mechanism is also pointed out. The conclusions are summarized in section 8 , and the appendix contains a brief discussion of gauge invariant variables and related zero mode issues.

\section{Preliminaries}

In this section we introduce the brane induced gravity model and describe our setup for its analysis: the use of gauge invariant variables, description of thick branes in terms of a density profile and its use as a regulator. Finally we consider the bulk-brane relations and the appearance of 4 new gauge invariant variables peculiar to the bulk-brane setup.

\subsection{The model and its origins}

In brane induced gravity models, the Universe is regarded as a 3-brane with coordinates $\sigma^{\mu}(\mu=0, \cdots, 3)$ embedded in a non-compact $(d=4+n)$-dimensional bulk spacetime with coordinates $x^{M}$ through $x^{M}(\sigma)$. In the basic model, dynamics of gravity is governed by an Einstein-Hilbert action $S_{E H}^{b u l k}$ for the bulk metric $G_{M N}(x)$ and a similar action $S_{E H}^{b r a n e}$ for the brane induced metric, $g_{\mu \nu}=\partial_{\mu} x^{M} \partial_{\nu} x^{N} G_{M N}$, sourced by brane matter $S_{m}^{\text {brane }}$ [1], 2]. In this paper, we also allow for adding a brane tension (brane cosmological constant) term $S_{\Lambda}^{b r a n e}$ and extrinsic curvature corrections $S_{\Omega}^{\text {brane }}$, to be specified later. Then ${ }^{6}$,

$$
S=-A \int d^{d} x \sqrt{-G} R^{(d)}-B \int d^{4} \sigma \sqrt{-g} R^{(4)}+S_{\Omega}^{\text {brane }}+S_{\Lambda}^{\text {brane }}+S_{m}^{\text {brane }} .
$$

As argued in [1, 2, 17], such models naturally arise in braneworld setups as a result of integrating out massive matter on the brane following [42]. More specifically, they were shown to arise in non-supersymmetric string theory, as a result of closed string scattering off D-branes [18, 19] where the action (2.1), including $S_{\Omega}^{\text {brane }}$ can be computed explicitly ${ }^{7}$.

\footnotetext{
${ }^{6}$ We follow the sign conventions of Weinberg in 41 .

${ }^{7}$ The calculation of [18, 19] leads to the expected behaviour of $A \sim g_{s}^{-2}$ and $B \sim g_{s}^{-1}$ with the string coupling $g_{s}$. Mass dimensions are supplied by the string tension, $1 / \alpha^{\prime}$. A curious feature of their result is that while, $A>0$, one gets $B<0$. Also, the relative sign between $S_{E H}^{b r a n e}$ and $S_{\Omega}^{\text {brane }}$ is opposite to what appears in the Gauss-Codazzi equation. However, for the BIG results to be relevant to phenomenology, we must take $B>0$ and adjust the parameter values appropriately, as will be specified later.
} 
At this order in the $\alpha^{\prime}$ perturbation theory, there are also bulk $R^{2}$ terms, in the GaussBonnet combination, that do not contribute to our analysis, as well as terms involving other massless string states that are ignored here. A different string theory realization was proposed in [20]. Here for the couplings $A, B$, etc. we do not use their calculated high energy values, but treat them as low energy phenomenological parameters.

The $d=5$ model is the well known DGP model that has been extensively investigated. As argued in [5, 6] it cannot address the problem of the observed smallness of cosmological constant. $d=6$ is also special and was investigated in [24, 25, 26]. Below, we are interested mainly in $d>6$ models.

\subsection{Flat background and gauge invariant variables}

We investigate brane induced gravity at the linearized level around a flat background, which is technically the easiest and a good first approximation. Consider a flat bulk with a flat brane and split $\left\{x^{M}\right\}$ into $\left\{x_{\|}^{\mu}, x_{\perp}^{i}\right\}$, respectively, parallel and perpendicular to the brane. The flat brane corresponds to $x_{\|}^{\mu}=\sigma^{\mu}, x_{\perp}^{i}=y_{0}^{i}$ (constants). Linearizing $G_{M N}(x)$, $g_{\mu \nu}(\sigma)$ and $x^{M}(\sigma)$ around this background gives the fluctuations $H_{M N}, h_{\mu \nu}, f^{\mu}$ and $y^{i}$,

$$
\begin{aligned}
& G_{M N}=\eta_{M N}+H_{M N}(x), \quad g_{\mu \nu}=\eta_{\mu \nu}+h_{\mu \nu}(\sigma), \\
& x_{\|}^{\mu}(\sigma)=\sigma^{\mu}+f^{\mu}(\sigma), \quad x_{\perp}^{i}(\sigma)=y_{0}^{i}+y^{i}(\sigma) .
\end{aligned}
$$

To first order, $h(x(\sigma))=h\left(x_{\|}\right)$, etc., and the fluctuations are related by the linearized pullback equation $h_{\mu \nu}\left(x_{\|}\right)=H_{\mu \nu}\left(x_{\|}, y_{0}\right)+\partial_{\mu} f_{\nu}+\partial_{\nu} f_{\mu}$. Infinitesimal bulk and brane diffeomorphisms transform the fluctuations as,

$$
\begin{aligned}
& \delta x^{M}=\xi^{M}: \quad \delta_{\xi} H_{M N}=-2 \partial_{(M} \xi_{N)}, \quad \delta_{\xi} h_{\mu \nu}=0, \quad \delta_{\xi} f^{\mu}=\xi^{\mu}, \\
& \delta \sigma^{\mu}=\lambda^{\mu}: \quad \delta_{\lambda} H_{M N}=0, \quad \delta_{\lambda} h_{\mu \nu}=-2 \partial_{(\mu} \lambda_{\nu)}, \delta_{\lambda} f^{\mu}=-\lambda^{\mu} .
\end{aligned}
$$

The last equation arises since $x^{M}(\sigma)$ are scalars under brane diffeomorphism so that $\delta_{\lambda} f^{\mu} \equiv$ $\delta_{\lambda} x^{\mu}=-\lambda^{\nu} \delta_{\nu}^{\mu}$. The $f_{\mu}$ play a role similar to Stückelberg fields in massive gravity.

To solve the equations of motion, we use gauge invariant variables instead of gauge fixing transformations $(2.4)^{8}$. This disentangles the spin 0 and spin 2 modes of the graviton and promotes $f_{\mu}$ to new gauge invariant variables. The bulk field $H_{M N}$ decomposes as

$$
H_{M N}=H_{M N}^{\perp}+\partial_{M} A_{N}+\partial_{N} A_{M}+\partial_{M} \partial_{N} \Phi+\frac{1}{d} \eta_{M N} S
$$

where $\partial^{M} H_{M N}^{\perp}=0, H_{M}^{\perp M}=0$, and $\partial^{M} A_{M}=0$. Then, $H_{M N}^{\perp}$ and $S$ are gauge invariant while $A_{M}$ and $\Phi$ give rise to the transformations of $H_{M N}$ in (2.4). The relevant projection operators are listed in appendix A. The bulk Einstein-Hilbert action becomes,

$$
\begin{aligned}
S_{E H}^{b u l k} & =-\frac{A}{4} \int d^{d} x\left(\partial^{L} H^{M N} \partial_{L} H_{M N}-\partial^{L} H \partial_{L} H+2 \partial_{L} H \partial_{N} H^{L N}-2 \partial_{M} H^{M N} \partial^{L} H_{L N}\right) \\
& =-\frac{A}{4} \int d^{d} x\left[\partial^{L} H^{\perp M N} \partial_{L} H_{M N}^{\perp}-\frac{(d-2)(d-1)}{d^{2}} \partial^{L} S \partial_{L} S\right]
\end{aligned}
$$

\footnotetext{
${ }^{8}$ Customarily, one fixes static (Monge) gauge, $f^{\mu}=0$, on the $x^{\mu}(\sigma)$ and harmonic gauge on $H_{M N}$.
} 
where gauge dependent terms drop out. To obtain the contribution to the equation of motion from the second line, express the variations $\delta H_{N M}^{\perp}$ and $\delta S$ in terms of $\delta H_{M N}$ using the projection operators in Appendix A. The linearized Einstein-Hilbert operator becomes,

$$
\left(\mathcal{E}_{d} H\right)_{M N}=\frac{1}{2} \square_{d} H_{M N}^{\perp}+\frac{d-2}{2 d}\left(\partial_{M} \partial_{N}-\eta_{M N} \square_{d}\right) S .
$$

Similarly, the induced brane field $h_{\mu \nu}$ is decomposed as

$$
h_{\mu \nu}=h_{\mu \nu}^{\perp}+\partial_{\mu} a_{\nu}+\partial_{\nu} a_{\mu}+\partial_{\mu} \partial_{\nu} \phi+\frac{1}{4} \eta_{\mu \nu} s
$$

where $\partial^{\mu} h_{\mu \nu}^{\perp}=0, h_{\mu}^{\perp \mu}=0$ and $\partial^{\mu} a_{\mu}=0 . h_{\mu \nu}^{\perp}$ and $s$ are gauge invariant. The brane Einstein-Hilbert action $S_{E H}^{\text {brane }}$ and operator $\left(\mathcal{E}_{4} h\right)_{\mu \nu}$ can be read off from (2.6) and (2.7) for $d=4$, after appropriate field replacements. There are also new gauge invariant variables based on $f^{\mu}$ and $y^{i}$ to be introduced later in equations 2.17 and 5.7.

\subsection{Thick branes and "blurred" quantities}

Solving the equations of motion involves the massless scalar propagator in $d$ dimensions,

$$
G\left(x_{\|}-x_{\|}^{\prime}, x_{\perp}-x_{\perp}^{\prime}\right)=-\int d^{4} k \int d^{n} q \frac{e^{i k\left(x_{\|}-x_{\|}^{\prime}\right)+i q\left(x_{\perp}-x_{\perp}^{\prime}\right)}}{k^{2}+q^{2}-i \epsilon} .
$$

$k_{\mu}$ and $q_{i}$ denote momenta parallel and transverse to the brane. For two points restricted to lie on a zero thickness brane, $x_{\perp}^{i}=x_{\perp}^{i}=y_{0}^{i}$ and the $q$ integral in $G\left(x_{\|}-x_{\|}^{\prime}, 0\right)$ diverges for $n>1$. Correspondingly, a bulk field $S$ sourced by a brane localized source $\delta\left(x_{\perp}-y_{0}\right) T\left(x_{\|}\right)$, i.e., $S\left(x_{\|}, x_{\perp}-y_{0}\right)=\int d^{4} x_{\|}^{\prime} G\left(x_{\|}-x_{\|}^{\prime}, x_{\perp}-y_{0}\right) T\left(x_{\|}\right)$also diverges as $x_{\perp} \rightarrow y_{0}$. Hence, the restriction of such bulk fields to the brane is ill defined. Essentially, a zero thickness brane is a point source in transverse directions leading to a $\left|x_{\perp}-y_{0}\right|^{-(n-2)}$ divergence, as in the Coulomb or Yukawa potentials.

This problem is resolved by realizing that dynamics give branes a form factor and hence an effective thickness, as discussed in [43] for D-branes and in [17] for solitonic branes. This is taken into account by replacing $\delta\left(x_{\perp}-y_{0}\right)$ by a brane thickness profile function, $P\left(x_{\perp}-y_{0}\right)$, with normalization $\int d^{n} x_{\perp} P=1$. Then, the divergent brane restriction $S\left(x_{\|}, 0\right)$ of the bulk field is replaced by the "blurred" field $\langle S\rangle\left(x_{\|}\right)$, a weighted average over the brane width,

$$
\langle S\rangle\left(x_{\|}\right)=\int d^{n} x_{\perp} P\left(x_{\perp}-y_{0}\right) S\left(x_{\|}, x_{\perp}-y_{0}\right),
$$

With Fourier transform conventions $P\left(x_{\perp}-y_{0}\right)=\int d^{n} q \widetilde{P}(q) e^{i q\left(x_{\perp}-y_{0}\right)}$, one gets the momentum space equation

$$
\langle\widetilde{S}\rangle(k)=(2 \pi)^{n} \int d^{n} q \widetilde{P}(q) \widetilde{S}(k, q) .
$$

In the same way, the divergent brane restricted propagator $G\left(x_{\|}-x_{\|}^{\prime}, 0\right)$ gets replaced by its well defined thick brane analogue,

$$
\langle G\rangle\left(x_{\|}-x_{\|}^{\prime}\right)=\int d^{n} x_{\perp} d^{n} x_{\perp}^{\prime} P\left(x_{\perp}-y_{0}\right) G\left(x_{\|}-x_{\|}^{\prime}, x_{\perp}-x_{\perp}^{\prime}\right) P\left(x_{\perp}^{\prime}-y_{0}\right) .
$$


Physically, this is the propagation amplitude from $x_{\|}$to $x_{\|}^{\prime}$ located on the brane, with a small delocalization in the transverse directions. This does not yet take into account physical effects due to the presence of the brane. However, this construct will contribute to the physical propagators and the interesting physics associated with it will be discussed in the next section.

The corresponding momentum space expression (with $\widetilde{P}$ depending only on $q^{2}$ ) is

$$
\langle\widetilde{G}\rangle(k)=-(2 \pi)^{n} \int d^{n} q \frac{[\widetilde{P}(q)]^{2}}{k^{2}+q^{2}-i \epsilon} .
$$

Again, for a zero-thickness brane, $\widetilde{P}=1$ and the expression diverges for $n>1$. For thick branes, $\widetilde{P}(q)$ effectively implements a UV regularization at high $q$ through a length scale associated with the brane width, $\omega$. Earlier, a non-zero brane width has been used to justify implementing a sharp cutoff $\sim 1 / \omega$ on the $q$ integral [23, 27], or solving the equations separately outside and inside the thick brane [17, 34]. Here, we will explicitly retain a smooth profile function $\widetilde{P}$ through which the results depend on the brane width $\omega$. Such dependences are not affected by the actual form of $P$, that encodes high energy effects and which will only change the numerics.

Since the normalized $P$ has mass dimension $n, \widetilde{P}(q)$ is dimensionless. Then rotational invariance in the transverse space means it only depends on the combination $q^{2} \omega^{2}$. This implies (after simple manipulations in (2.12)),

$$
\langle\widetilde{G}\rangle(k)=\frac{1}{\omega^{n-2}} \Sigma^{-1}\left(\omega^{2} k^{2}\right),
$$

depending on $k$ only through $u=\omega^{2} k^{2}$. The expression captures the form of divergence as $\omega \rightarrow 0$. Later, $\Sigma$ will appear in the same way as a self-energy correction in quantum field theory, hence the notation. Note that $w^{n}\langle\widetilde{G}\rangle$ has the correct dimension for a propagator.

The use of the brane effective width $\omega$ means that the theory is valid for describing interactions of transverse gravitons with the brane as long as $q<1 / \omega$. Probing the brane at shorter scales is meaningless in the low energy theory. To insure that interactions too cannot probe beyond the brane width, the effective theory must also be restricted to $u=(k \omega)^{2}<1$, for momenta along the brane.

For the most part we do not need the functional form of $P$. But for explicit calculations, a natural choice, motivated by [20], is the Gaussian form ${ }^{9}$,

$$
P\left(x_{\perp}\right)=\frac{1}{(\omega \sqrt{2 \pi})^{n}} e^{-\left(x_{\perp} / 2 \omega\right)^{2}}, \quad \widetilde{P}(q)=\frac{1}{(2 \pi)^{n}} e^{-q^{2} \omega^{2} / 2} .
$$

The profile function can also be discussed in a covariant setup, but that is not needed here.

\subsection{Bulk-brane relations}

For a thick brane, the pullback equation relating bulk and brane metric fluctuations becomes,

$$
h_{\mu \nu}=\left\langle H_{\mu \nu}\right\rangle+\partial_{\mu} f_{\nu}+\partial_{\nu} f_{\mu},
$$

\footnotetext{
${ }^{9}$ In contrast with a sharp cut-off on $q$, a Gaussian $\widetilde{P}(q)$ leads to a non-negative $P\left(x_{\perp}-y_{0}\right)$, consistent with its interpretation as a thickness profile. The main results are not affected by these choices.
} 
with only $x_{\|}$dependences. Note that fields intrinsically defined on the brane are not affected by the "blurring" procedure (2.10). Then, using (2.5) and (2.8) gives,

$$
\left\langle H^{\perp}\right\rangle_{\mu \nu}=h_{\mu \nu}^{\perp}-\partial_{\mu} F_{\nu}-\partial_{\nu} F_{\mu}-\eta_{\mu \nu}\left(\frac{1}{d}\langle S\rangle-\frac{1}{4} s\right) .
$$

The $F^{\mu}$ are new variables invariant under both bulk and brane gauge transformations,

$$
F_{\mu}=f_{\mu}+\left\langle A_{\mu}\right\rangle-a_{\mu}+\frac{1}{2} \partial_{\mu}(\langle\Phi\rangle-\phi) .
$$

The gauge dependent variables in $F_{\mu}$ do not appear in the action (2.1). However, solving the equations of motion requires either gauge fixing or using gauge invariant variables along with (2.16). Thus, in bulk-brane setups, gauge variant variables survive through $F_{\mu}$ and contribute to the solutions for the brane fields $s$ and $h^{\perp}$. The implication of this as a broken phase realization of 4-dimensional general covariance is discussed in the last section.

\section{The Basic Brane Induced Gravity Model}

In this section, we review and further investigate the solutions of the basic BIG model 11, 2] based on bulk and brane Einstein-Hilbert actions of (2.1) but without $S_{\Lambda}^{\text {brane }}$ and $S_{\Omega}^{\text {brane }}$. First, we solve the linearized equations of motion for the brane fields and review the tachyon/ghost problem [28]. The propagator is then analyzed in depth, extracting a mass, a decay width and a modulation of the gravitational coupling. The suppression of graviton decay into the bulk is explained with the help of the optical theorem. The reader not interested in the details of solving the equations of motion can directly jump to the solutions (3.15) and (3.16).

\subsection{Equation of motion and solutions}

For the basic BIG model, the linearized equation of motion for $H_{M N}$ expressed in terms of Einstein-Hilbert operators (2.7) and for thick branes, takes the form

$$
A\left(\mathcal{E}_{d} H\right)^{M N}+P\left(x_{\perp}-y_{0}\right)\left(B\left(\mathcal{E}_{4} h\right)^{\mu \nu}+\frac{1}{2} T^{\mu \nu}\right) \delta_{\mu}^{M} \delta_{\nu}^{N}=0 .
$$

In our momentum space conventions, it becomes ${ }^{10}\left(\right.$ with $\left.p^{M}=\left\{k^{\mu}, q^{i}\right\}\right)$,

$$
\begin{aligned}
-A\left[\left(k^{2}+q^{2}\right)\right. & \left.\widetilde{H}^{\perp M N}+\frac{d-2}{d}\left(p^{M} p^{N}-\eta^{M N}\left(k^{2}+q^{2}\right)\right) \widetilde{S}\right]_{(k, q)} \\
& -\widetilde{P}(q)\left[B\left(k^{2} \widetilde{h}^{\perp \mu \nu}+\frac{1}{2}\left(k^{\mu} k^{\nu}-\eta^{\mu \nu} k^{2}\right) \widetilde{s}\right)-\widetilde{T}^{\mu \nu}\right]_{(k)} \delta_{\mu}^{M} \delta_{\nu}^{N}=0 .
\end{aligned}
$$

This is to be combined with the surface equation (2.16). In the following, the $i \epsilon$ terms in the bulk propagator are not always written explicitly, but are finally included in the blurred propagator (2.12).

\footnotetext{
${ }^{10}$ The $(\mu \nu)$ components alone can determine the brane fields, but it is more convenient to use all equations.
} 
The $\eta_{M N}$-trace of (3.2) determines $\widetilde{S}$ in terms of $\widetilde{s}$,

$$
\widetilde{S}(k, q)=-\frac{d}{A(d-1)(d-2)} \frac{\widetilde{P}(q)}{k^{2}+q^{2}-i \epsilon}\left(\widetilde{T}_{\mu}^{\mu}+\frac{3}{2} B k^{2} \widetilde{s}\right)_{(k)} .
$$

Restricting to the brane by using (2.11) and the expression for $\langle\widetilde{G}\rangle$ in (2.12), one gets,

$$
\langle\widetilde{S}\rangle(k)=\frac{d}{A(d-1)(d-2)}\langle\widetilde{G}\rangle\left(\widetilde{T}_{\mu}^{\mu}+\frac{3}{2} B k^{2} \widetilde{s}\right)_{(k)} .
$$

For later convenience, we express $\widetilde{S}(k, q)$ in terms of $\langle\widetilde{S}\rangle(k)$,

$$
\widetilde{S}(k, q)=-\frac{\widetilde{P}(q)}{k^{2}+q^{2}} \frac{1}{\langle\widetilde{G}\rangle}\langle\widetilde{S}\rangle .
$$

The $(\mu, i)$ components give (since "blurring" now involves integrating over an odd function of $q^{i}$ ),

$$
\widetilde{H}^{\perp \mu i}(k, q)=-\frac{d-2}{d} \frac{k^{\mu} q^{i}}{k^{2}+q^{2}} \widetilde{S}(k, q), \quad \Rightarrow \quad\left\langle\widetilde{H}^{\perp}\right\rangle^{\mu i}(k)=0 .
$$

However, note that using (3.5), and the transversality of $H^{\perp}$, one gets,

$$
\left\langle q_{i} \widetilde{H}^{\perp \mu i}\right\rangle(k)=-k_{\nu}\left\langle\widetilde{H}^{\perp}\right\rangle^{\mu \nu}=\frac{d-2}{d} \frac{\langle\widetilde{g}\rangle}{\langle\widetilde{G}\rangle} k^{\mu}\langle\widetilde{S}\rangle .
$$

where we introduce a new function $\langle\widetilde{g}\rangle(k)$ (that will drop out of most expressions),

$$
\langle\widetilde{g}\rangle=(2 \pi)^{n} \int d^{n} q \frac{q^{2} \widetilde{P}^{2}}{\left(k^{2}+q^{2}\right)^{2}} .
$$

The $(i, j)$ components give,

$$
\widetilde{H}^{\perp i j}(k, q)=-\frac{d-2}{d}\left(\frac{q^{i} q^{j}}{k^{2}+q^{2}}-\eta^{i j}\right) \widetilde{S}(k, q) .
$$

From this one can immediately see that $\left\langle q_{i} \widetilde{H}^{\perp i j}\right\rangle=0$ and, for $i \neq j,\left\langle\widetilde{H}^{\perp i j}\right\rangle=0$. However,

$$
\left\langle\widetilde{H}_{j}^{\perp j}\right\rangle(k)=\frac{d-2}{d}\left(\frac{\langle\widetilde{g}\rangle}{\langle\widetilde{G}\rangle}+n\right)\langle\widetilde{S}\rangle, \quad\left\langle q_{i} q_{j} \widetilde{H}^{\perp i j}\right\rangle(k)=-\frac{d-2}{d} k^{2} \frac{\langle\widetilde{g}\rangle}{\langle\widetilde{G}\rangle}\langle\widetilde{S}\rangle .
$$

The surface equation (2.16), on taking a trace and a double divergence, gives

$$
\left\langle\widetilde{H}_{\mu}^{\perp \mu}\right\rangle=-2 i k^{\mu} \widetilde{F}_{\mu}-\frac{4}{d}\langle\widetilde{S}\rangle+\widetilde{s}, \quad k^{\mu} k^{\nu}\left\langle\widetilde{H}^{\perp}\right\rangle_{\mu \nu}=-k^{2}\left(2 i k^{\mu} \widetilde{F}_{\mu}+\frac{1}{d}\langle\widetilde{S}\rangle-\frac{1}{4} \widetilde{s}\right) .
$$

Since $\left\langle\widetilde{H}_{\mu}^{\perp \mu}\right\rangle=-\left\langle\widetilde{H}_{i}^{\perp i}\right\rangle$ and $k^{\mu} k^{\nu}\left\langle\widetilde{H}^{\perp}\right\rangle_{\mu \nu}=\left\langle q_{i} q_{j} \widetilde{H}^{\perp i j}\right\rangle(k)$, on using (3.10), 3.11) become,

$$
2 i k^{\mu} F_{\mu}=\left[(d-2)\left(\frac{\langle\widetilde{g}\rangle}{\langle\widetilde{G}\rangle}+n\right)-4\right] \frac{\langle\widetilde{S}\rangle}{d}+\widetilde{s}, \quad 2 i k^{\mu} F_{\mu}=\left[(d-2) \frac{\langle\widetilde{g}\rangle}{\langle\widetilde{G}\rangle}-1\right] \frac{\langle\widetilde{S}\rangle}{d}+\frac{\widetilde{s}}{4} \text {. }
$$


Eliminating $k_{\mu} \widetilde{F}^{\mu}$ gives a relation between $\langle\widetilde{S}\rangle$ and $\widetilde{s}$ as,

$$
\frac{(d-1)(d-5)}{d}\langle\widetilde{S}\rangle=-\frac{3}{4} \widetilde{s} .
$$

Substituting back in the expression for $k_{\mu} \widetilde{F}^{\mu}$ determines it in terms of $\widetilde{s}$. Combining this with the divergence of (2.16) and then using (3.7) leads to the solution for the $\widetilde{F}^{\mu}$ fields,

$$
\widetilde{F}^{\mu}=-\frac{i}{4} \frac{k^{\mu}}{k^{2}} U \widetilde{s}, \quad \text { where, } \quad U=\frac{1}{2} \frac{d-2}{(d-5)(d-1)}\left(n-3 \frac{\langle\widetilde{g}\rangle}{\langle\widetilde{G}\rangle}\right) .
$$

Finally, combining (3.13) with (3.4) gives the solution (3.15) below for $\widetilde{s}$ in terms of $T=T_{\mu}^{\mu}$.

The $(\mu, \nu)$ Components of (3.2) can be solved for $H_{\mu \nu}^{\perp}$. One can then compute $\left\langle\widetilde{H}_{\mu \nu}^{\perp}\right\rangle$ using (3.5). Using (2.16), this becomes an equation for $\widetilde{h}_{\mu \nu}^{\perp}$ in terms of $\widetilde{S}, \widetilde{s}$ and $F^{\mu}$ all of which are known in terms of $\widetilde{T}_{\mu \nu}$. Thus one has the final solutions,

$$
\begin{gathered}
\widetilde{s}(k)=-\frac{2}{3 B} \frac{1}{k^{2}+\frac{A}{B} \frac{d-2}{2(d-5)}\langle\widetilde{G}\rangle^{-1}} \widetilde{T} \\
\widetilde{h}_{\mu \nu}^{\perp}=\frac{1}{B} \frac{1}{k^{2}-\frac{A}{B}\langle\widetilde{G}\rangle^{-1}}\left(\widetilde{T}_{\mu \nu}-\frac{1}{3}\left(\eta_{\mu \nu}-\frac{k^{\mu} k^{\nu}}{k^{2}}\right) \widetilde{T}\right) .
\end{gathered}
$$

From this one can directly read off the gauge independent parts of the Greens functions $G_{\mu \nu \mu^{\prime} \nu^{\prime}}^{(0,2)}$, or the 4-dimensional gauge invariant amplitude,

$$
\begin{aligned}
& \int d^{4} x \int d^{4} x^{\prime} T^{\mu \nu}(x) G_{\mu \nu \mu^{\prime} \nu^{\prime}}\left(x-x^{\prime}\right) T^{\mu^{\prime} \nu^{\prime}}\left(x^{\prime}\right) \\
&=\int d^{4} x T^{\mu \nu}(x) h_{\mu \nu}(x)=\int d^{4} x\left(T^{\mu \nu} h_{\mu \nu}^{\perp}+\frac{1}{4} T s\right) .
\end{aligned}
$$

If needed, the $h_{\mu \nu}$ in a given gauge can be constructed by adding gauge transformations, $h_{\mu \nu}=h_{\mu \nu}^{\perp}+\frac{1}{4} \eta_{\mu \nu} s+\partial_{\mu} \xi_{\nu}+\partial_{\nu} \xi_{\mu}$, and solving the gauge conditions for the $\xi_{\mu}$.

One can immediately draw a number of conclusions, mostly known in the literature, based on the structure of $s$ and $h^{\perp}$ solutions:

(1) Zero thickness limit: Note that in the absence of extra dimensions, $\langle\widetilde{G}\rangle_{n=0}=$ $-1 / k^{2}$, leading to the solutions for ordinary Einstein-Hilbert gravity,

$$
\left(\widetilde{h}_{\mu \nu}^{\perp}\right)_{(n=0)}=\frac{1}{A^{\prime}} \frac{1}{k^{2}}\left(\widetilde{T}_{\mu \nu}-\frac{1}{3}\left(\eta_{\mu \nu}-\frac{k^{\mu} k^{\nu}}{k^{2}}\right) \widetilde{T}\right), \quad(\widetilde{s})_{n=0}=-\frac{2}{3 A^{\prime}} \frac{1}{k^{2}} \widetilde{T} .
$$

Also $\langle\widetilde{g}\rangle_{n=0}=0$ gives $\left(\widetilde{F}^{\mu}\right)_{n=0}=0$. The BIG results 3.15$),(3.16)$ differ from this only in the $\langle\widetilde{G}\rangle^{-1}$ dependent terms. Now, while the brane width $\omega$ was introduced to keep $\langle\widetilde{G}\rangle$ finite for $n>1$, the final solution is well defined for $\omega \rightarrow 0$. Then, $\langle\widetilde{G}\rangle$ diverges (from (2.13)), resulting again in (3.18). Hence, for $n>2$, modifications of 4-dimensional gravity arise only for thick branes. Since the spin 2 and spin 0 parts of the propagator in BIG have the same coefficients as in ordinary gravity (except $d=5$ where $\widetilde{s}=0$ ), there is no vDVZ discontinuity 31, 32, 33 in the $\omega \rightarrow 0$ limit. 
(2) Tachyon problem: For $n>2,\langle\widetilde{G}\rangle\left(k^{2}=0\right)$ is finite and contributes a hard mass to the propagators. The scale of the mass is set by $A \omega^{n-2} / B$ and the gravitational coupling is given by $G_{N} \sim 1 / B$. Thus, for phenomenological reasons, $B$ should be large and $A \omega^{n-2}$ should be small. But $\langle\widetilde{G}\rangle^{-1}$ appears with opposite signs in the denominators, indicating that if $h^{\perp}$ has a healthy mass, then $s$ will be tachyonic or vice versa [28]. For the conventional choice of $B>0$ and $A>0, h^{\perp}$ cannot have a tachyonic pole (since for any $k^{2}>0$, (2.12) gives $\left.\langle\widetilde{G}\rangle(k)<0\right)$. Then, $s$ is tachyonic.

3) Ghost problem: It is well known [31, 44, 45] that a propagator for $h_{\mu \nu}$ with the tensor structure of ordinary massless gravity in 4-dimensions, is ghost free only for zero graviton masses. Thus the non-zero masses and the $-2 / 3$ factor in the $\widetilde{s}$ solution makes the tachyonic spin 0 mode also a ghost [28]. The presence of this tachyonic ghost in brane induced gravity has so far hampered its further development. One of our purposes in this paper is to shed light on the origin of this ghost mode.

4) Unstable gravitons: Ignoring the tachyonic ghost $\widetilde{s}$ field, the massive spin 2 graviton $h^{\perp}$ on the brane is not a stable particle but a resonance state. Technically, this is because $\langle\widetilde{G}\rangle$ has a branch cut from $k^{2}=0$ to $-\infty$ due to the continuum of KaluzaKlein modes resulting from an uncompactified bulk $([5,[6])$. For very small masses, the life time is very large. In the next subsection we will explain the origin of the decay and its suppression using standard quantum field theory and then carry out a detailed analysis of the propagator.

5) String theory limit: The string theory computation of the BIG action in [18, 19] obtained $B<0$, making $h^{\perp}$ the tachyonic ghost. But in this setup, in terms of the string coupling $g_{s}, A \sim 1 / g_{s}^{2}$ and $B \sim 1 / g_{s}$. Assuming $\omega \sim g_{s}^{0}$, weak string coupling corresponds to large masses, beyond the validity limits of the theory. Even for D3-branes where $n=6$ and $\omega^{4} \sim g_{s}$ is a more natural choice, masses are $g_{s}$-independent and at string scale. In either case, for $k^{2}$ values within the validity range of the theory, the propagator is given by $-\langle\widetilde{G}\rangle(k) / A$, avoiding the tachyon/ghost problems, and gravity is essentially higher dimensional. However, the orbifold based construction of 20] that attempts to derive BIG from string theory with the phenomenologically interesting parameter ranges, does not evade the tachyon/ghost problem in this manner.

In the rest of this section we concentrate on the healthy spin 2 mode, returning to the origins of the ghost mode in the next section.

\subsection{Graviton decay from optical theorem}

A main feature of BIG, analyzed around flat background, is that $h^{\perp}$ is a massive unstable graviton in 4 dimensions due to the possibility of gravitons escaping into the bulk [1, 6, 6]. However, the graviton lifetime must be large for phenomenological reasons. Below, we show that the suppression of graviton decay can be explained using general field theory arguments. The detailed structure of the propagator is analyzed in the next subsection.

The bulk propagator $G\left(x-x^{\prime}\right)$ (2.9) gives the amplitude for graviton propagation from any $x$ to any $x^{\prime}$ (ignoring the tensor structure and for canonically normalized fields, so no $1 / A$ factor $)$. But $\langle G\rangle\left(x_{\|}-x_{\|}^{\prime}\right)$ is constructed to describe a restricted propagation between two points with their $x_{\perp}$ coordinates delocalized over the width $\omega$ of a thin region 
that would be occupied by the brane. Of course, there is a finite probability $\sigma_{\text {escape }}$ that gravitons emitted at $x_{\|}$within the thin region, end up somewhere in the bulk. Such processes are not described by the restricted propagator $\langle G\rangle$ and appear to it as decay channels, thus giving it an imaginary part. The relationship is quantified by the optical theorem where, in terms of the corresponding amputated 2-point function $\omega^{-n}\langle\widetilde{G}\rangle^{-1}$ (with $\omega$ factors restoring the correct dimensions for the propagator),

$$
2 \operatorname{Im}\left(\omega^{-n}\langle\widetilde{G}\rangle^{-1}\right) \sim \sigma_{\text {escape }} \neq 0 .
$$

This is a geometric result that holds for any region of space that would be occupied by a thick brane and applies to gravitons not yet dynamically affected by a physical brane.

Now, with a physical brane in place and after taking gravitational dynamics on the brane into account, we obtained a brane-to-brane propagator $\widetilde{G}_{b b}=-\left(k^{2}-\frac{A}{B}\langle\widetilde{G}\rangle^{-1}\right)^{-1}$ in (3.16), but now written for the canonically normalized brane field and with suppressed tensor structure. The stability of the corresponding state is again encoded in the imaginary part of the amputated 2-point function which now gives,

$$
2 \operatorname{Im} \widetilde{G}_{b b}^{-1}=2 \frac{A}{B} \operatorname{Im}\langle\widetilde{G}\rangle^{-1} \sim \frac{A \omega^{n}}{B} \sigma_{\text {escape }} .
$$

Hence, graviton decay on the brane is still due to escape into the bulk, but with an amplitude suppressed by a factor $A \omega^{n} / B$ as compared to the unhindered escape in the absence of brane gravitational dynamics. This is a way of seeing that a large brane Einstein-Hilbert term confines gravitons to the brane by suppressing their escape into the bulk.

Finally, for completeness, one can also compute the brane-to-bulk propagator,

$$
\widetilde{G}_{B b}(k, q)=\frac{-\widetilde{P}(q)\langle\widetilde{G}\rangle^{-1}}{k^{2}+q^{2}}\left[\frac{1}{B} \frac{1}{k^{2}-\frac{A}{B}\langle\widetilde{G}\rangle^{-1}}\right] .
$$

The brane-to-bulk decay probability $\sigma_{\text {brane } \rightarrow \text { bulk }}$ can be constructed out of this after proper amputations (using $G_{b b}$ for the brane leg and $G$ for the bulk leg). One can then verify the optical theorem for the physical propagator,

$$
2 \operatorname{Im} \widetilde{G}_{b b}^{-1} \sim \sigma_{\text {brane } \rightarrow \text { bulk }}
$$

In this sense, the verification of the optical theorem on the brane requires using the braneto-bulk propagator, since the brane theory alone is not unitary.

\subsection{Graviton mass, decay width and coupling}

Let us investigate the properties of the spin 2 graviton $h^{\perp}(3.16)$ in more detail. The physics of the corresponding brane-to-brane propagator follows from the structure of $\langle\widetilde{G}\rangle\left(k^{2}-i \epsilon\right)$. We will see that the propagator has the standard form for a massive unstable particle in QFT. More importantly, for small masses it is amenable to the standard particle physics approximation methods.

To evaluate $\langle\widetilde{G}\rangle(2.12)$ we use the Gaussian form (2.14) for $\widetilde{P}(q)$. Doing the angular integrals in $q$-space gives,

$$
\langle\widetilde{G}\rangle=-2 N \int d q q^{n-1} \frac{e^{-q^{2} \omega^{2}}}{k^{2}+q^{2}-i \epsilon}, \quad N^{-1}=(4 \pi)^{\frac{n}{2}} \Gamma\left(\frac{n}{2}\right) .
$$


First, let us consider the even $n$ case and set $2 m=n-2$ (the result for odd $n$ is given later). After some manipulations,

$$
\langle\widetilde{G}\rangle(k)=-\frac{(-1)^{m} N}{\omega^{n-2}} u^{m}\left(\frac{\partial}{\partial u}\right)^{m}\left(e^{u} E_{1}(u-i \epsilon)\right),
$$

where $u=\omega^{2} k^{2}$ and we have used the notation 46],

$$
E_{1}(u-i \epsilon)=\int_{u-i \epsilon}^{\infty} d t \frac{e^{-t}}{t}
$$

Since $\partial_{u} E_{1}(u)=-e^{u} / u$, for $\Sigma^{-1}=\omega^{n-2}\langle\widetilde{G}\rangle$ one gets

$$
\Sigma^{-1}(u-i \epsilon)=-(-1)^{m} N\left[u^{m} e^{u} E_{1}(u-i \epsilon)+\sum_{r=1}^{m}(-1)^{r}(r-1) ! u^{m-r}\right] .
$$

The corresponding expression for odd $n$ is given by equation (3.35) below.

Remember that the $i \epsilon$ prescription tells us how to handle the $k^{2}+q^{2}=0$ poles in (2.12). Now, after performing the $q$-integrations, the same prescription will dictate the correct $\epsilon \rightarrow 0$ limit. To see what happens, note that the function $E_{1}(z)$ is the analytic continuation, to the complex plane, of the exponential integral Ei(-u) defined, for real $u$, by

$$
E i(-u)=-\int_{u}^{\infty} d t \frac{e^{-t}}{t}
$$

$E i(-u)$ is defined over the entire real line, but $E_{1}(z)$ has a branch cut from $z=0$ to $z=-\infty$ along the negative real axis. The crucial point is that as $\epsilon \rightarrow 0$, we approach the branch cut from below and

$$
\lim _{\epsilon \rightarrow 0} E_{1}(u-i \epsilon)=-E_{i}(-u)+i \pi \theta(-u)
$$

where, $\theta(-u)$ is the unit step function. The imaginary part is the origin of the complex pole in the propagator, and hence of graviton decay ${ }^{11}$. Writing $\Sigma^{-1}=a+i b$, the real and imaginary parts are given by

$$
\begin{aligned}
& a(u)=(-1)^{m} N\left[u^{m} e^{u} \operatorname{Ei}(-u)-\sum_{r=1}^{m}(-1)^{r}(r-1) ! u^{m-r}\right], \\
& b(u)=-\pi N|u|^{m} e^{-|u|} \theta(-u) .
\end{aligned}
$$

Finally, in terms of $\Sigma=\Sigma_{1}+i \Sigma_{2}$, the brane-to-brane propagator in the $h^{\perp}$ solution (3.16) is (with canonical field normalization and suppressed tensor structure),

$$
\widetilde{G}_{b b}=\frac{-1}{k^{2}-\frac{A \omega^{n-2}}{B}\left[\Sigma_{1}\left(\omega^{2} k^{2}\right)+i \Sigma_{2}\left(\omega^{2} k^{2}\right)\right]},
$$

\footnotetext{
${ }^{11}$ Approaching the branch cut from above (corresponding to $E_{1}(u+i \epsilon)$ ), would give $-i \pi$ which does not lead to decay. So the correct sign is dictated by the $i \epsilon$ prescription in the bulk propagator.
} 
The above analysis then gives,

$$
\Sigma_{1}(u)=\frac{a}{a^{2}+b^{2}}, \quad \Sigma_{2}(u)=\frac{-b}{a^{2}+b^{2}} \geq 0 .
$$

This propagator has the familiar quantum field theory form where the analogue of $\Sigma$ arises due to self-energy corrections. It has a complex mass pole and, strictly speaking, the graviton mass and decay width are given by the real and imaginary parts of the complex pole. In practice, determining the location of the complex pole is not easy. But since the determination of mass anyway becomes somewhat arbitrary due to the finite life-time, an approximation can be made provided, near the mass pole, $\left|\Sigma_{1}\right|$ is much larger than $\left|\Sigma_{2}\right|$. In such cases, a mass can be defined as the pole position with only $\Sigma_{1}$ present, while $\Sigma_{2}$ gives the decay width (see, for example, 47, 48]). This is often a valid approximation in perturbative quantum field theory. Fortunately, one can show that it also holds here for small enough masses: For $m \geq 1$, as $u \rightarrow 0, \Sigma_{2}$ vanishes as $u^{m}$ while $\Sigma_{1}$ goes to a constant $-1 /[N(m-1) !]$. Hence, for a small enough mass, at the mass pole $M^{2}=-u / \omega^{2}$, one has $\left|\Sigma_{1}\right|>>\left|\Sigma_{2}\right|$, as wished.

Then, in this approximation the mass $M^{2}=-k^{2}$ is determined, on ignoring $\Sigma_{2}$, by

$$
\left[\frac{B}{A \omega^{n}}\right] \omega^{2} k^{2}=\Sigma_{1}\left(\omega^{2} k^{2}\right)
$$

For $n>2, \Sigma_{1}(0)=-[N(m-1) !]^{-1}<0$. So, by continuity, $\Sigma_{1}\left(\omega^{2} k^{2}\right)<0$ for small arguments in the neighborhood of zero. Then the graviton is non-tachyonic, $M^{2}>0$. More concretely, this is visible in Fig.1(a) where the right hand side of the above equation is plotted against $u=\omega^{2} k^{2}$ for $n=6,5, \cdots, 3$ (odd $n$ curves are based on (3.35)) and the left hand side is plotted for two different slopes $B / A \omega^{n}$, with values chosen only for illustrative purposes. The intersections determine the masses $M^{2}=-u / \omega^{2}$, with larger slopes resulting in smaller masses. The plots also show that $\Sigma_{1}$ is monotonic for $n>2$ and the propagator has a single mass pole. In Fig.1(b) we plot $\Sigma_{2}$ vs $\omega^{2} k^{2}$. Comparing curves for the same $n$ in both plots again shows that for very small masses, $\left|\Sigma_{2}\left(-\omega^{2} M^{2}\right)\right|<<\left|\Sigma_{1}\left(-\omega^{2} M^{2}\right)\right|$, justifying the approximation (the curves for $n=1,2$ look rather different and are not shown here).

Now, in terms of the mass as determined above, the propagator has the standard form,

$$
\widetilde{G}_{b b}=-\frac{Z(k)}{k^{2}+M^{2}-i \frac{A \omega^{n-2}}{B} Z(k) \Sigma_{2}(k)},
$$

where,

$$
Z^{-1}(k)=1-\frac{\left(A \omega^{n-2} / B\right) \Sigma_{1}\left(\omega^{2} k^{2}\right)+M^{2}}{k^{2}+M^{2}} .
$$

This will look more familiar when $\Sigma_{1}(u)$ is Taylor expanded around $u=-\omega^{2} M^{2}$.

Note that $Z$ retains a momentum dependence which amounts to a momentum dependence of the effective gravitational coupling,

$$
G_{N}^{e f f}(k)=Z(k) / B
$$




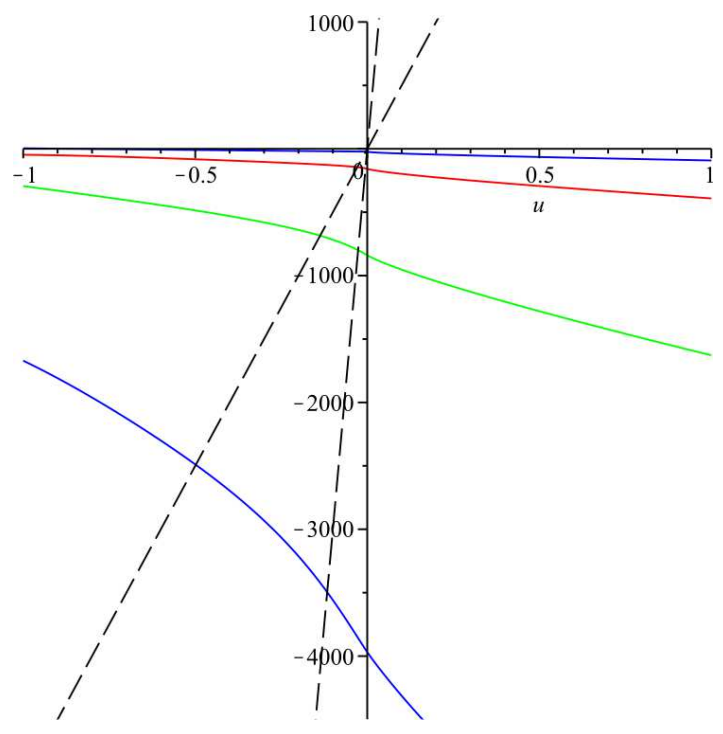

(a) Determination of Mass

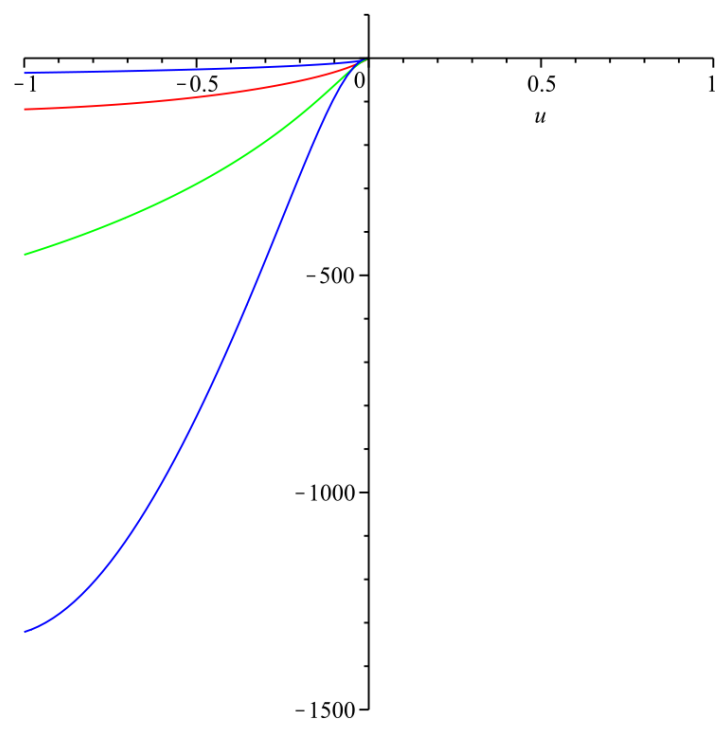

(b) Decay Width

Figure 1: Behaviour of $\Sigma_{1}(u)$ (left) and $\Sigma_{2}(u)$ (right) for codimensions $n=6$ (the lowest curves) through $n=3$ (close to the $x$-axis)

This momentum dependence can be expressed entirely in terms of the dimensionless parameters $u$ and $A \omega^{n} / B$. In particular, for time independent sources $\left(k^{2}>0\right), \Sigma_{2}=0$ and this is the only modification besides the graviton mass.

A simple analysis of $Z(k)$ shows that at large momenta, $\left|k^{2}\right|>>M^{2}, Z \sim 1$. But for small momenta, $\left|k^{2}\right| \sim M^{2}$, on Taylor expanding around the mass pole given by (3.30), one gets $Z<1$. Hence the gravitational coupling is weaker at large distances, but the variation is not drastic. This is over and above the effects due to graviton mass and decay width.

Now, comparing (3.31) to the relativistic Breit-Wigner form near the mass pole, the decay width is identified as

$$
\Gamma=\left.\frac{A \omega^{n-2}}{M B} Z(u) \Sigma_{2}(u)\right|_{u=-\omega^{2} M^{2}}=-\left.M Z(u) \frac{\Sigma_{2}(u)}{\Sigma_{1}(u)}\right|_{u=-\omega^{2} M^{2}}>0
$$

Once again, note that it is the $i \epsilon$ prescription in the bulk propagator that leads to the correct sign for the decay width.

For odd $n$ the analysis proceeds along similar lines, except that the exponential integral is replaced by the error function and, with $2 m^{\prime}=n-1$, we get,

$$
\begin{aligned}
\Sigma^{-1}(u-i \epsilon)=-(-1)^{m^{\prime}} N \pi\left[u^{m^{\prime}-\frac{1}{2}} e^{u}\right. & (1-\operatorname{erf}(\sqrt{u-i \epsilon})) \\
& \left.+\frac{1}{\sqrt{\pi}} \sum_{r=1}^{m^{\prime}}(-1)^{r} \frac{(2 r-3) ! !}{2^{r-1}} u^{m^{\prime}-r}\right] .
\end{aligned}
$$


In fact, this expression has been used to plot the curves for $n=3,5$ in Fig.1(a) and Fig.1(b). A unified description of both even and odd $n$ is given in terms of the Whittaker functions,

$$
\Sigma^{-1}=-\frac{u^{\frac{n}{4}-1} e^{\frac{1}{2} u}}{(4 \pi)^{\frac{n}{2}}} W_{-\frac{n}{4}, \frac{n-2}{4}}(u-i \epsilon) .
$$

For completeness, in Fig.2, we show a representative plot for the spectral density function,

$$
\rho(s)=-\frac{1}{\pi} \operatorname{Im} G_{b b}\left(k^{2}=-s\right),
$$

to show that it too has the standard form for an unstable particle, again with the $A \omega^{n} / B$ value chosen only for the purpose

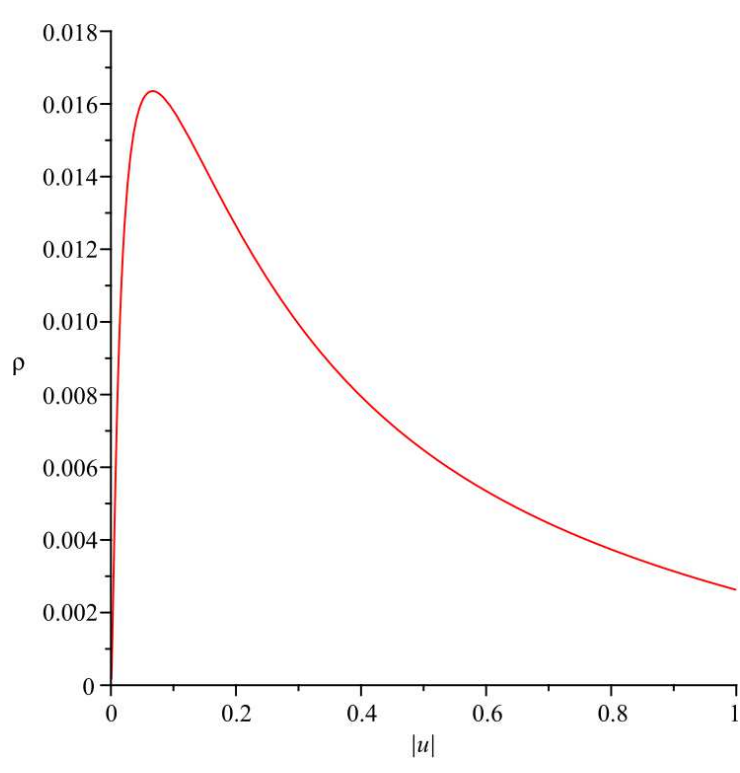

Figure 2: A Sample Spectral Density Function of illustration.

To summarize, we have shown that although the origin of the "self-energy" contribution, $\Sigma$, to the propagator in the BIG setup is very different from its origin in perturbative quantum field theory, the resulting unstable massive particles behave in very similar ways and are amenable to the same approximation. Hence, the modification of the spin 2 graviton propagator $(3.16)$, contained in $\langle\widetilde{G}\rangle^{-1}$, breaks up into 3 contributions: a hard mass $M$ given by (3.30), a decay width $\Gamma$ (3.34) and a momentum dependent Newton "constant" (3.33).

\section{Screening of $\Lambda$ and the Origin of Tachyon/Ghost Problem}

The possible resolution of the cosmological constant problem is the main reason for interest in $n>2$ brane induced gravity, despite its ghost/tachyon issues. Support for this expectation comes, as discussed in [5, 6], from the classical solutions of $d$-dimensional gravity [14, 15] that describe a 3-brane with an ADM mass density corresponding to a non-zero brane tension (which is the same as the brane cosmological constant). To be precise, the classical solutions describe this setup at distances far away from the location of the brane core. The interpretation is that, for codimensions $n>2$, a brane cosmological constant $\Lambda$ can curve directions transverse to the brane rather than those parallel to it, keeping the brane metric flat ${ }^{12}$. Thus the effect of $\Lambda$ is absorbed by bulk curvature and it does not curve the brane worldvolume to a de Sitter spacetime.

Now, the presence of a brane Einstein-Hilbert term in the action does not affect this classical solution. But for a large enough coefficient, it is expected to dictate the dynamics of metric perturbations $h_{\mu \nu}$ around the classical solution. This is why one expects $n>2$

\footnotetext{
12 5, 6] also consider time dependent solutions but these do not show up in our linearized analysis and are not discussed here.
} 
BIG models to describe effectively 4-dimensional gravity with suppressed $\Lambda$ (The $n=2$ model is somewhat special and has been analyzed in some detail in [24, 25, 26]).

Unfortunately, implementing the above scenario is not as straightforward. In reality, to study metric perturbations $h_{\mu \nu}$, one needs classical solutions that are valid in the vicinity of the brane (at least with some effective thickness included). The existing solution [14, 15] is not adequate for this purpose since the energy momentum tensor that sources it near the brane core, does not correspond to a brane $\Lambda$ source.

Hence, in practice, in BIG with codimension $n>2$, the analysis of metric perturbations has mostly been performed in a flat background, instead of one sourced by $\Lambda$ (even though the latter is used to argue for the suppression of $\Lambda$ ). It is also in the flat background analysis that one encounters the tachyonic ghost problem reviewed in the previous section. One may conjecture that this problem is an artifact of the flat approximation and would go away once a full fledged analysis is performed around a curved background sourced by $\Lambda$. However, in the absence of a classical solution adequate for such analysis, here we point out that the flat background analysis of the previous section is, by itself, sufficient to study the response of gravity to a small brane cosmological constant and exhibit its suppression. This also has ramifications for what to expect from a curved background analysis for the resolution of the ghost issue (as will be detailed below). The healthy spin 2 part of the graviton, on which we have concentrated so far, is not relevant here. Below we return to the unhealthy scalar mode to find that,

- The "tachyonic" mass is actually healthy and is instrumental in filtering out $\Lambda$ in the flat background approximation. It arises, at least partly, as an artifact of the flat approximation and as a substitute for the background curvature.

- For phenomenologically interesting values of parameters (corresponding to a small graviton mass), the ghost nature is more intractable and will most likely persist even when metric perturbations are analyzed in the background curved by $\Lambda$, unless one ends up with massless gravity on the brane in a fully non-linear background.

- The 4-dimensional Newton constant and other couplings are modified by the screened $\Lambda$, consistent with [3, 5, 6]. This may help in avoiding a very low bulk gravity scale $A$, but may introduce hierarchy problems for other couplings.

- Finally we emphasize that BIG and massive Fierz-Pauli gravity (as studied in [38]) use different mechanisms to respond to $\Lambda$, in spite of a superficial similarity.

\subsection{Filtering out of brane cosmological constant}

It is easy to see that brane induced gravity for $n>2$ is not very sensitive to a brane cosmological constant: The addition of the brane tension/cosmological constant term, $S_{\Lambda}=-\Lambda \int d^{4} \sigma \sqrt{-g(X(\sigma))}$, to the action amounts, at the linearized level, to the shift

$$
\widetilde{T}_{\mu \nu}(k) \rightarrow \widetilde{T}_{\mu \nu}^{\prime}(k)=\widetilde{T}_{\mu \nu}(k)+\Lambda \eta_{\mu \nu} \delta^{(4)}(k) .
$$

The validity of the perturbative analysis around flat background requires that $\Lambda$ is small, of the same order as $T_{\mu \nu}$. In ordinary Einstein-Hilbert gravity, $\Lambda$, however small, destabilizes 
the flat space solution toward a de Sitter space (the manifestation of this at the propagator level is described below). Hence, in a modified theory of gravity the stability of flat space in the presence of $\Lambda$ is an indication that gravity has been made less sensitive to the cosmological constant. The analogue of this argument for Fierz-Pauli massive gravity is considered in [38], although the actual mechanisms differ.

Let us write the solutions (3.15) and (3.16) explicitly in terms scalar and tracelesstransverse components of $T_{\mu \nu}$,

$$
\widetilde{s}(k)=-\frac{2}{3 B} \frac{1}{k^{2}+\frac{A}{B} \frac{d-2}{2(d-5)}\langle\widetilde{G}\rangle^{-1}} \widetilde{T}_{s}, \quad \widetilde{h}_{\mu \nu}^{\perp}=\frac{1}{B} \frac{1}{k^{2}-\frac{A}{B}\langle\widetilde{G}\rangle^{-1}} \widetilde{T}_{\mu \nu}^{\perp} .
$$

As discussed in Appendix A, it is only in this form that the solutions are valid for a cosmological constant source. For such a source, $\widetilde{T}_{\Lambda \mu \nu}^{\perp}=0$ and $\widetilde{T}_{\Lambda s}=4 \Lambda \delta^{(4)}(k)$, leading to $\widetilde{h}_{\Lambda \mu \nu}^{\perp}=0$ and,

$$
\widetilde{s}_{\Lambda}(k)=4 c \delta^{(4)}(k), \quad \text { where } \quad c=-\frac{4}{3} \frac{d-5}{d-2} \frac{\Lambda}{A}\langle\widetilde{G}\rangle(0) .
$$

The crucial point is that only for $n>2,\langle\widetilde{G}\rangle(0)$ is finite and the analysis well defined (for example, for a Gaussian profile and for even $\left.n,\langle\widetilde{G}\rangle(0)=-\left(\frac{n}{2}-2\right) ! /\left[(4 \pi)^{\frac{n}{2}} \Gamma\left(\frac{n}{2}\right) \omega^{n-2}\right]\right)$. Thus, in coordinate space, $\Lambda$ effectively shifts the background from $s=0$ to a constant value $s=4 c$, keeping the flat background essentially unchanged. It is instructive to contrast this with Einstein-Hilbert gravity where one would get $\widetilde{s}_{\Lambda} \sim \Lambda \delta^{(4)}(k) / k^{2}$, which is a solution to $\square_{4} s_{\Lambda} \sim \Lambda$. Then $s_{\Lambda}$ is quadratic in distance and diverges at large distances, indicating that flat space is not a good starting point for a perturbative expansion of de Sitter space. In contrast, in BIG, $\Lambda$ does not produce a non-flat metric on the brane and is hence filtered out ${ }^{13}$.

The mechanism by which BIG filters out $\Lambda$ is sometimes heuristically explained on the basis of the behaviour of the $n=1$ model in which gravity becomes higher dimensional, and hence weaker, at very large distances. But this model does not filter out $\Lambda$ and the $n>2$ model that does, has a different large distance behaviour. So let us reiterate two relevant aspects of the above analysis:

1. Only a non-zero $\langle\widetilde{G}\rangle(0)$ is relevant to filtering out $\Lambda$. The momentum dependence of $\langle\widetilde{G}\rangle$ (that contributes to graviton decay and a varying gravitational coupling) does not play any role in this, in contrast to the more general "filter" mechanisms of [37]

2. It also follows that at large distances $\left(k^{2} \rightarrow 0\right)$, the behaviour of gravity does not become higher dimensional, unlike the $n=1$ DGP model. Hence the filtering of the cosmological constant cannot be attributed to such a behaviour.

In BIG, the cosmological constant is completely filtered out at the linearized level. One needs to go beyond the linear analysis to make a statement about its remnant effects. In particular, explaining the small observed value of $\Lambda$ (or dark energy density) is a different issue not addressed by BIG at this stage.

\footnotetext{
${ }^{13}$ Codimensions $n=1,2$ give $\langle\widetilde{G}\rangle(0)=\infty$ due to an IR divergent $q$-integral and the above discussion of filtering, formulated around flat background, does not apply. For the the $n=2$ case, see [24, 25, 26].
} 


\subsection{On the origin of the tachyon and ghost problems}

As we have seen, a tachyonic ghost mode is encountered while analyzing BIG around flat background 28]. Although flat space is a valid solution to the $\Lambda=0$ model, one may still suspect that the tachyon and ghost problems are related to this very set up. After all, a generic 3-brane always has a tension which, in some sense, is the stuff that holds the brane together. So one always has to work with the full action,

$$
S_{E H}^{b u l k}+S_{E H}^{b r a n e}+S_{\Lambda}^{\text {brane }}+S_{m}^{\text {brane }} .
$$

Even though a $\Lambda \rightarrow 0$ limit may exist, in that limit $S_{\Lambda}^{\text {brane }}=\Lambda \int d^{4} \sigma \sqrt{-g}$ should be replaced by the action for a tensionless brane [49, 50] rather than totally eliminated. But tensionless branes have special properties that make them unsuitable for our purposes and hence need not be considered. This line of argument tells us that the "basic" brane induced gravity model reviewed in section 3 does not really correspond to a brane setup, unless augmented by a brane tension term. Could this also provide a solution to the tachyon and ghost problems? This possibility is discussed below.

One approach to addressing this question is to consider, as suggested in [3, 5, 6], a large $S_{\Lambda}^{\text {brane }}$ so the matter action $S_{m}^{\text {brane }}$ can be neglected to first approximation. This leads to classical solutions for bulk gravity sourced by $\Lambda$. Around this one should study metric perturbations sourced by $S_{m}^{b r a n e}$ and influenced by $S_{E H}^{b r a n e}$. As pointed out above, the existing solutions [14, 15] are not adequate for this purpose since they do not satisfy the equations of motion with the correct brane source. Hence, such analysis has not yet been carried out (see footnote 3 ).

However, the above parameter range $\left(S_{\Lambda}^{\text {brane }}>>S_{m}^{\text {brane }}\right)$ is not the only one to explore. In fact, we saw that the linearized analysis is already capable of exploring the model in the regime where $S_{\Lambda}^{\text {brane }}$ and $S_{m}^{\text {brane }}$ have comparable but small contributions. Thus, it can also provide some insight into the implication of the brane tension for the tachyon and ghost problems.

The tachyon and ghost problems showed up in the propagator (3.15) for the $s$ field which has the general structure

$$
-\frac{2}{3} \frac{1}{B k^{2}-A \omega^{n-2}\left|N^{(0)}\right| \Sigma(k)} .
$$

The overall sign signals a ghost and the relative sign in the denominator, a tachyon. The appearance of $A$ and $B$ coefficients (associated with bulk and brane actions) shows that $\Sigma(k)$ (relevant to the tachyonic nature) arises entirely from integrating out bulk modes, while the rest (the $-2 / 3$ factor and hence the ghost nature) originates in the brane Einstein-Hilbert term. Let's emphasize that the above form is a valid way of presenting and interpreting the propagator as long as the $B$ term in the action dominates over the $A$ term. This is the phenomenologically interesting case, with a very light graviton and effectively 4-dimensional gravity. It is also in this range that the tachyon and ghost identifications hold ${ }^{14}$.

\footnotetext{
${ }^{14}$ For example, in the opposite case when the $A$ term dominates over the $B$ term (as in string theory setups), the propagation is described by the $\Sigma(k)$ term and gravity is healthy and essentially $d$-dimensional.
} 
- The tachyon problem: Now, this is not hard to dismiss. In the previous subsection we saw that the "tachyonic" mass of $s$ (more precisely, the $\Sigma(0) \neq 0$ part) was instrumental in "absorbing" $\Lambda$ and keeping the brane metric flat. On the other hand, the classical solutions of [14, 15] indicate that $\Lambda$ can equally well be absorbed by curving directions transverse to the brane, keeping the brane metric flat. The comparison indicates that approximating the bulk as flat, has to force a mass on $s$ in order for the brane metric to remain flat in the presence of $\Lambda$. In this sense, the mass is at least partly an artifact of the flat background approximation and is functionally equivalent to a "healthy" bulk curvature to be sourced by $\Lambda$. This demonstrates that the "tachyonic" mass is not an unhealthy feature. Indeed, $m_{s}^{2}$ is tachyonic only with respect to the ghost-like kinetic term of $s$, and as such has a positive contribution to the $s$ field Hamiltonian $\sim \int\left(-\dot{s}^{2}-\nabla s^{2}+m_{s}^{2} s^{2}\right)$.

- The ghost problem: This however is more fundamental and may not be cured even if the flat background is replaced by a curved one sourced by the brane tension. The reason is that even in such a curved background, the brane metric remains flat and hence its fluctuations are described by $S_{E H}^{b r a n e}$, expanded around flat 4-dimensional spacetime. This contributes a factor $-(2 / 3)\left(1 / B k^{2}\right)$ to the scalar mode propagator, exactly as in flat background. A modified "self-energy" term, $\Sigma_{\text {curved }}$, arises from integrating out bulk modes, but now it also depends on the brane tension. As before, small graviton masses and effectively 4-dimensional gravity require a large $B$ coefficient. Thus the overall propagator again has the standard 4-dimensional tensor structure, corrected by self-energy terms. Now, it is well known that a propagator with a tensor structure associated with the 4-dimensional Einstein-Hilbert action is ghost free only in the massless limit (where the scalar mode ghost cancels against a contribution from the spin 2 mode). As soon as the mass poles are shifted away from zero, the scalar mode ghost remains uncanceled.

An implication is that the ghost can be avoided only if $\Sigma_{\text {curved }}(0)=0$. In a flat space background, this would have prevented the theory from filtering out the cosmological constant. But in a curved background, where $\Lambda$ is already absorbed in the background curvature, this could lead to a consistent theory of massless gravity with filtered out $\Lambda$. However, it is not obvious that a background sourced by the brane tension will lead to such a self-energy contribution. In fact the opposite seems to be the case by a continuity argument: In the small $\Lambda$ limit, the expressions should reduce to what we have already computed, in which case $\Sigma(0) \neq 0$. Hence we conclude that in the phenomenologically interesting parameter ranges, the scalar ghost will be a generic feature of brane induced gravity in any background, except those for which $\Sigma_{\text {curved }}(0)=0$.

The absence of a ghost in the $d=5$ DGP model is a numeric coincidence. On general grounds, reduction of gravity from $d$ to 4 dimensions gives a factor $d-5$ in the scalar sector. In the BIG setup, this results in solutions with $s=0$, thus evading the ghost problem.

\subsection{Implication for couplings}

The structure of the solution (4.3) essentially corresponds to a warped metric with its 
standard implications for the couplings. The metric in the presence of $\Lambda$ and matter sources becomes,

$$
g_{\mu \nu}(x)=(1+c) \eta_{\mu \nu}+h_{\mu \nu}^{(m)}(x)
$$

where $c \sim \Lambda /\left(A \omega^{n-2}\right)>0$ and $h_{\mu \nu}^{(m)}$ is the metric perturbation sourced by matter. Adopting the standard convention to use $\eta_{M N}$ as the flat spacetime metric, one defines the physical metric

$$
g_{\mu \nu}^{\prime}=\frac{g_{\mu \nu}}{(1+c)} .
$$

In terms of this, $B \int \sqrt{g} R=(1+c) B \int \sqrt{g}^{\prime} R^{\prime}$. For the matter action, $\int \sqrt{g} \mathcal{L}(g, \psi, \lambda)=$ $(1+c)^{2} \int \sqrt{g}^{\prime} \mathcal{L}\left(g^{\prime}, \psi^{\prime}, \lambda^{\prime}\right)$, where the matter fields, collectively denoted by $\psi$, and their couplings $\lambda$, have to be scaled appropriately for the equivalence principle to hold. This results in an effective $B^{\prime}=B /(1+c)$ or an effective Newton constant $G_{N}^{\prime}=(1+c) G_{N}$, consistent with related observations in [17, 5, 6, 3, 25, 26]. Although this analysis is valid for small $c$ (so that our perturbative treatment remains valid) it indicates that for a large $\Lambda$ one needs a starting $B$ larger than the observed $M_{p}^{2}$ (for $c>0$ ). This can relax the constraint on the bulk coupling $A$ based on the smallness of the graviton mass by a factor $(1+c)$ which is welcome news. However, it may also introduce new hierarchy issues for other standard model couplings.

\subsection{Contrast with Fierz-Pauli Massive gravity}

In the ghost-free FP massive gravity theory around flat background, reviewed in a later section, the spin-2 graviton $h^{\perp}$ has a mass $m_{2}$, whereas, to avoid the ghost, the mass $m_{0}$ of the scalar mode $s$ is sent to infinity. Hence for any matter source $T_{\mu \nu}, s=0$. The caveat is that a constraint equation one gets for $s$ (for $m_{0}=\infty$ ) only implies $\square_{4} s=0$ so, in general, $s=c_{1}+c_{2} s^{\text {harmonic }}$. Substituting back into the equations, gives $c_{2}=0$ for any source and $c_{1}=-4 \Lambda G_{N} / 3 m_{2}^{2} \neq 0$ only for a cosmological constant source. To contrast the two theories, in BIG, $s$ is always sourced and remains well behaved in the presence of $\Lambda$ due to its own mass, but in the ghost free FP gravity, $s$ is zero except for a $\Lambda$ source and the screening parameter is the mass of spin- 2 graviton $m_{2}$, which is itself not sourced by $\Lambda$ ! A FP theory with finite $m_{0}$ (and hence a ghost), when sourced by $\Lambda$, would behave similar to BIG for $n>2$, with $m_{0}$ as the screening parameter. However, the $m_{0} \rightarrow \infty$ limit is not continuous. Thus, although superficially similar, BIG with $n>2$ and FP massive gravity use different mechanisms to filter out $\Lambda$.

\section{Inclusion of Extrinsic Curvature Terms}

We now solve the modified equations of motion after the inclusion of the extrinsic curvature terms. These are ubiquitous in any brane induced gravity setup and arise at the same perturbative order as the induced Einstein-Hilbert term 18, 19, 52. The brane transverse fluctuations enter as new degrees of freedom. The brane tension can be included in $T_{\mu \nu}$. Again the analysis is performed in terms of gauge invariant variables. It turns out that the extrinsic curvature terms have no effect whatsoever on the solutions for the brane fields. While this does not cure the tachyon/ghost problem, it at least insures that 4-dimensional 
gravity is not modified in other unwanted ways by the extrinsic curvature terms. The negative result is essentially due to the $Z_{2}$ reflection symmetry of the background about the brane position. Breaking this symmetry will lead to extra non-trivial contributions from the extrinsic curvature terms. With this summary, the reader not interested in the details of the calculation can safely skip this section.

\subsection{Extrinsic curvature term in the action}

The contribution of the extrinsic curvature to the action is 18

$$
S_{\Omega}=C \int d^{4} \sigma \sqrt{-g}\left(\Omega_{\alpha \beta}^{M} \Omega_{M}^{\alpha \beta}-\Omega_{\alpha}^{M \alpha} \Omega_{M \beta}^{\beta}\right)
$$

where

$$
\Omega_{\alpha \beta}^{M}=\partial_{\alpha} \partial_{\beta} x^{M}-\gamma_{\alpha \beta}^{\lambda} \partial_{\lambda} x^{M}+\Gamma_{N K}^{M} \partial_{\alpha} x^{N} \partial_{\beta} x^{K} .
$$

Here $\gamma$ and $\Gamma$ are the Christoffel connections corresponding to the metrics $g_{\alpha \beta}(x(\sigma))$ and $G_{M N}(x(\sigma))$, respectively. For a thin brane the quadratic action for the fluctuations can be worked out by setting $g=\eta+h, G=\eta+H$ and $\delta X^{M}=\left\{f^{\mu}, y^{i}\right\}$,

$$
\begin{aligned}
S_{\Omega}=C \int & d x_{\|}^{4}\left[\frac{1}{2}\left(\partial_{\alpha} H_{i \beta} \partial^{\alpha} H^{i \beta}-\partial_{\alpha} H^{\alpha i} \partial^{\beta} H_{\beta i}\right)+\partial_{\alpha} H^{\alpha i} \partial_{i} H_{\beta}^{\beta}-\partial_{\alpha} H^{i \beta} \partial_{i} H^{\alpha \beta}\right. \\
+ & \left.\frac{1}{4}\left(\partial_{i} H_{\alpha \beta} \partial^{i} H^{\alpha \beta}-\partial_{i} H_{\alpha}^{\alpha} \partial^{i} H_{\beta}^{\beta}\right)+y^{i} \partial_{i}\left(\square_{4} H_{\alpha}^{\alpha}-\partial_{\alpha} \partial_{\beta} H^{\alpha \beta}\right)+\cdots\right],
\end{aligned}
$$

where the ellipsis stand for functions of $h_{\mu \nu}-H_{\mu \nu}$ that do not contribute to the equation of motion for a metric variation $\delta h_{\mu \nu}=\delta H_{\mu \nu}$.

As described in section 2, for thick branes, $H_{M N}(x(\sigma))$ are replaced by $\left\langle H_{M N}\right\rangle\left(x_{\|}\right)$. A new feature of $S_{\Omega}$ is that it contains transverse derivatives of $H$ restricted to the brane, $\left.\partial_{i} H\right|_{\text {brane }} \equiv\left(\frac{\partial}{\partial x_{\perp}^{i}} H\right)\left(x_{\|}, x_{\perp}^{i}=y_{0}^{i}\right)$. For a thick brane, these should be replaced by $\left\langle\partial_{i} H\right\rangle \equiv$ $\left\langle\frac{\partial}{\partial x_{\perp}^{i}} H\right\rangle\left(x_{\|}\right)$. The $y^{i}\left(x_{\|}\right)$are brane fields and do not need any "blurring". Hence, for thick branes, the appropriate action is,

$$
\begin{aligned}
S_{\Omega}=C \int & d x^{4}\left[\frac{1}{2}\left(\partial_{\alpha}\langle H\rangle_{i \beta} \partial^{\alpha}\langle H\rangle^{i \beta}-\partial_{\alpha}\langle H\rangle^{\alpha i} \partial^{\beta}\langle H\rangle_{\beta i}\right)+\partial_{\alpha}\langle H\rangle^{\alpha i}\left\langle\partial_{i} H\right\rangle_{\beta}^{\beta}\right. \\
& -\partial_{\alpha}\langle H\rangle^{i \beta}\left\langle\partial_{i} H\right\rangle^{\alpha \beta}+\frac{1}{4}\left(\left\langle\partial_{i} H\right\rangle_{\alpha \beta}\left\langle\partial^{i} H\right\rangle^{\alpha \beta}-\left\langle\partial_{i} H\right\rangle_{\alpha}^{\alpha}\left\langle\partial^{i} H\right\rangle_{\beta}^{\beta}\right) \\
& \left.+y^{i}\left(\square_{4}\left\langle\partial_{i} H\right\rangle_{\alpha}^{\alpha}-\partial_{\alpha} \partial_{\beta}\left\langle\partial_{i} H\right\rangle^{\alpha \beta}\right)+\cdots\right] .
\end{aligned}
$$

\subsection{Equations of motion with extrinsic curvature contributions}

To obtain the contribution of $S_{\Omega}$ to the equations of motion for a thick brane, one considers variations $\delta y^{i}, \delta\langle H\rangle, \delta\left\langle\partial_{i} H\right\rangle$ and re-expresses the last two in terms of the bulk variation $\delta H$. In particular,

$$
\begin{aligned}
\delta\left\langle\partial_{i} H\right\rangle_{M N} & =\int d^{n} x_{\perp} P\left(x_{\perp}-y_{0}\right) \partial_{i} \delta H_{M N}\left(x_{\|}, x_{\perp}-y_{0}\right) \\
& =-\int d^{n} x_{\perp} \partial_{i} P\left(x_{\perp}-y_{0}\right) \delta H_{M N}\left(x_{\|}, x_{\perp}-y_{0}\right) .
\end{aligned}
$$


Then for $\delta H$ the variation can be written in a compact from as,

$$
\delta S_{\Omega}=-2 C \int d^{d} x\left[P\left(x_{\perp}-y_{0}\right) \partial_{\alpha} \mathcal{N}^{i \alpha \beta} \delta H_{i \beta}-\frac{1}{2} \partial_{i} P\left(x_{\perp}-y_{0}\right) \mathcal{N}^{i \alpha \beta} \delta H_{\alpha \beta}\right],
$$

where, to linear order,

$$
\begin{array}{r}
\mathcal{N}_{\alpha \beta}^{i}\left(x_{\|}\right)=\left\langle\Omega_{\alpha \beta}^{i}-\Omega_{\lambda}^{i \lambda} \eta_{\alpha \beta}\right\rangle=\partial_{\alpha} \partial_{\beta} y^{i}+\frac{1}{2}\left(\partial_{\alpha}\langle H\rangle_{\beta}^{i}+\partial_{\beta}\langle H\rangle_{\alpha}^{i}-\left\langle\partial^{i} H\right\rangle_{\alpha \beta}\right) \\
-\left(\square_{4} y^{i}+\partial_{\lambda}\langle H\rangle^{\lambda i}-\frac{1}{2}\left\langle\partial^{i} H\right\rangle_{\lambda}^{\lambda}\right) \eta_{\alpha \beta} \\
=\partial_{\alpha} \partial_{\beta} F^{i}+\frac{1}{2}\left(\partial_{\alpha}\left\langle H^{\perp}\right\rangle_{\beta}^{i}+\partial_{\beta}\left\langle H^{\perp}\right\rangle_{\alpha}^{i}-\left\langle\partial^{i} H^{\perp}\right\rangle_{\alpha \beta}\right) \\
-\left(\square_{4} F^{i}+\partial_{\lambda}\left\langle H^{\perp}\right\rangle^{\lambda i}-\frac{1}{2}\left\langle\partial^{i} H^{\perp}\right\rangle_{\lambda}^{\lambda}\right) \eta_{\alpha \beta}+\frac{3}{2 d} \eta_{\alpha \beta}\left\langle\partial^{i} S\right\rangle
\end{array}
$$

$F^{i}$ are gauge invariant extensions of $y^{i}$ (the analogues of $F^{\mu}$ in transverse directions),

$$
F^{i}=y^{i}+\left\langle A^{i}\right\rangle+\frac{1}{2}\left\langle\partial^{i} \Phi\right\rangle
$$

Now, from $\delta\left(S_{E H}^{b u l k}+S_{E H}^{b r a n e}+S_{\Omega}^{b r a n e}\right)$ one obtains the thick brane metric equation of motion with extrinsic curvature contributions contained in the $C$-dependent terms,

$$
\begin{gathered}
\frac{A}{2}\left[\square_{d} H^{\perp M N}+\frac{d-2}{d}\left(\partial^{M} \partial^{N}-\eta^{M N} \square_{d}\right) S\right] \\
+P\left(x_{\perp}-y_{0}\right)\left[\frac{B}{2}\left(\square_{4} h^{\perp \mu \nu}+\frac{1}{2}\left(\partial^{\mu} \partial^{\nu}-\eta^{\mu \nu} \square_{4}\right) s\right) \delta_{\mu}^{M} \delta_{\nu}^{N}-C \partial^{\lambda} \mathcal{N}_{\lambda \nu}^{i}\left(\delta_{i}^{M} \delta_{\nu}^{N}+\delta_{\nu}^{M} \delta_{i}^{N}\right)\right] \\
\quad+C \partial_{i} P\left(x_{\perp}-y_{0}\right) \mathcal{N}^{i \mu \nu} \delta_{\mu}^{M} \delta_{\nu}^{N}=-\frac{1}{2} P\left(x_{\perp}-y_{0}\right) T^{\mu \nu} \delta_{\mu}^{M} \delta_{\nu}^{N} .
\end{gathered}
$$

Also, in the quadratic action $S_{\Omega}$, the $y^{i}$ appear as Lagrange multipliers and their equation of motion is the constraint equation,

$$
\partial^{\mu} \partial^{\nu} \mathcal{N}_{\mu \nu}^{i} \equiv \frac{1}{2}\left\langle\partial^{i}\left(\square_{4} H_{\lambda}^{\perp \lambda}-\partial^{\mu} \partial^{\nu} H_{\mu \nu}^{\perp}+\frac{3}{d} \square_{4} S\right)\right\rangle=0
$$

But we will see that this is already contained in (5.8). The expression within braces is the scalar curvature of the metric $(\eta+H)_{\mu \nu}$.

\subsection{Solutions with extrinsic curvature contributions}

Below we show that the inclusion of $C$-dependent terms in (5.8) has no effect on the solutions for brane fields. We closely follow the steps in the $C=0$ case.

The $\eta_{M N}$-trace of (5.8) gives $S$ in terms of $s$ and $\mathcal{N}_{\mu \nu}^{i}$. On transforming to momentum space, one gets the solution

$$
\widetilde{S}(k, q)=\frac{-d}{A(d-1)(d-2)} \frac{\widetilde{P}(q)}{k^{2}+q^{2}}\left(\widetilde{T}_{\mu}^{\mu}+\frac{3}{2} B k^{2} \widetilde{s}+i 2 C q_{i} \widetilde{\mathcal{N}}_{\lambda}^{i \lambda}\right) .
$$


The relevant feature of this is that, since $\tilde{\mathcal{N}}_{\lambda}^{i \lambda}$ depends only on $k$, the $C$-dependent term is an odd function of $q^{i}$ and $\left\langle q_{i} \widetilde{\mathcal{N}}_{\lambda}^{i \lambda}\right\rangle=0$. Therefore, it does not contribute to $\langle\widetilde{S}\rangle$ which is, then, still given by (3.4). For later convenience, we record the analogue of (3.5),

$$
\widetilde{S}(k, q)=-\frac{\widetilde{P}(q)}{k^{2}+q^{2}}\left[\frac{\langle\widetilde{S}\rangle}{\langle\widetilde{G}\rangle}+i \frac{2 d}{A(d-1)(d-2)} C q_{i} \tilde{\mathcal{N}}_{\lambda}^{i \lambda}\right] .
$$

The $(i, j)$ Components of (5.8) are not affected by the $C$ dependent terms. Hence they yield again the solution (3.9) for $H_{i j}^{\perp}$ in terms of $S$. The solution (5.11) for $S$ then gives the same expressions for $\left\langle\widetilde{H}_{j}^{\perp j}\right\rangle$ and $\left\langle q^{i} q^{j} \widetilde{H}^{\perp i j}\right\rangle$ as in (3.10).

Manipulations of the surface equation also lead to the same result as the $C=0$ case. Hence $\langle S\rangle$ and $s$ are still related by (3.13) and finally the solution for $s$ is given by (3.15).

The $(\mu, i)$ Components of (5.8) contain a $C$-dependent term and give,

$$
\widetilde{H}^{\perp \mu i}(k, q)=-\frac{d-2}{d} \frac{k^{\mu} q^{i}}{k^{2}+q^{2}} \widetilde{S}(k, q)-2 i \frac{C}{A} \frac{\widetilde{P}}{k^{2}+q^{2}} k^{\lambda} \widetilde{\mathcal{N}}_{\lambda}^{i \mu} .
$$

However, $\left\langle q_{i} \widetilde{H}^{\perp \mu i}\right\rangle$ is still given by (3.7). Hence going through the same steps as before, we get the same solution (3.14) for $F_{\mu}$.

The $(\mu, \nu)$ Components of the equation of motion in momentum space is,

$$
\begin{aligned}
& \frac{A}{2}\left[\left(k^{2}+q^{2}\right) \widetilde{H}_{\mu \nu}^{\perp}+\frac{d-2}{2}\left(k_{\mu} k_{\nu}-\eta_{\mu \nu}\left(k^{2}+q^{2}\right)\right) \widetilde{S}\right] \\
& \quad+\widetilde{P}(q)\left[\frac{B}{2}\left(k^{2} \widetilde{h}_{\mu \nu}^{\perp}+\frac{1}{2}\left(k_{\mu} k_{\nu}-k^{2} \eta_{\mu \nu}\right) \widetilde{s}\right)-i C q_{i} \widetilde{\mathcal{N}}_{\mu \nu}^{i}(k)-\frac{1}{2} \widetilde{T}_{\mu \nu}\right]=0 .
\end{aligned}
$$

When we solve this for $H_{\mu \nu}^{\perp}$ and evaluate $\left\langle\widetilde{H}^{\perp}\right\rangle_{\mu \nu}$, the $C$-dependent term drops out (because of integration over an odd function of $q^{i}$ ). Using this to compute $\widetilde{h}_{\mu \nu}^{\perp}$ again gives the same result (3.16) as the $C=0$ case.

The $y^{i}$ Equation of motion (5.9) follows also as a consequence of the metric equation: contract (5.13) with $k^{\mu} k^{\nu}$, use $k^{\mu} k^{\nu} \widetilde{H}_{\mu \nu}^{\perp}=q^{i} q^{j} \widetilde{H}_{i j}^{\perp}$ and (3.9) to eliminate $\widetilde{H}_{i j}^{\perp}$ in favour of $\widetilde{S}$. This gives (5.9) in momentum space.

Thus, this constraint does not affect the solutions for the brane fields, but it provides an equation for $F^{i}$ as follows: Using $H_{\lambda}^{\perp \lambda}=-H_{i}^{\perp i}, \partial^{\mu} \partial^{\nu} H_{\mu \nu}^{\perp}=\partial^{i} \partial^{j} H_{i j}^{\perp}$ and (3.9) to rewrite these in terms of $S$, one gets

$$
k^{\mu} k^{\nu} \widetilde{\mathcal{N}}_{\mu \nu}^{i}=-\frac{i}{2 d}(d-1)(d-5) k^{2}\left\langle q^{i} \widetilde{S}\right\rangle=0
$$

Ignoring the 4-dimensional harmonic solutions, this gives $\left\langle q^{i} \widetilde{S}\right\rangle=0$, or $\left\langle\partial^{i} S\right\rangle=0$. Using the solution for $S$ in (5.10), this becomes (with no sum over $j$ ),

$$
\left\langle q^{j} \widetilde{S}\right\rangle=-i \frac{2 d C}{A(d-1)(d-2)}\left[\int d^{n} q \frac{\widetilde{P}^{2}(q)\left(q^{j}\right)^{2}}{k^{2}+q^{2}}\right]\left(\widetilde{\mathcal{N}}_{\lambda}^{j \lambda}\right)=0,
$$

which holds only if,

$$
\widetilde{\mathcal{N}}_{\lambda}^{j \lambda}(k) \equiv 3 k^{2} \widetilde{F}^{j}+3 i\left\langle q_{i} \widetilde{H}^{\perp j i}\right\rangle-i \frac{3}{2}\left\langle q^{j} \widetilde{H}_{i}^{\perp i}\right\rangle+i \frac{6}{d}\left\langle q^{j} \widetilde{S}\right\rangle=0 .
$$


Again, expressing $H_{i j}^{\perp}$ in terms of $S$ gives

$$
3 k^{2}\left(\widetilde{F}^{j}(k)+i \frac{d-2}{2 d}\left\langle\frac{q^{j} \widetilde{S}}{k^{2}+q^{2}}\right\rangle\right)=0 .
$$

But (5.11) implies $\left\langle q^{j} \widetilde{S} /\left(k^{2}+q^{2}\right)\right\rangle \propto \widetilde{\mathcal{N}}_{\lambda}^{j \lambda}=0$, so we finally get the $F^{i}$ equations,

$$
\square_{4} F^{j}=0 \text {. }
$$

Hence, up to harmonic functions, $F^{i}=0$. This is a consequence of the reflection symmetry of the set up in directions transverse to the brane. A background that breaks this symmetry can lead to non-trivial $F^{i}$. Note that for the DGP model with $d=5$, the constraint equation (5.14) is trivial and does not imply an equation for $F^{5}$.

Thus the presence of the extrinsic curvature related corrections has no effect on BIG at the linearized level, in particular not on the ghost problem. While this is discouraging, the positive aspect is that such terms (that on general grounds are ubiquitous in all braneworld models) do not cause large deviations from standard gravity.

\section{Revisiting Fierz-Pauli Massive Gravity}

In the remaining part of this paper, we turn to a comparison of brane induced gravity for $n>2$, with massive versions of Einstein-Hilbert gravity in 4 dimensions. In this section we start with a review Fierz-Pauli massive gravity theory [29, 30] (for reviews, see [53, 54]). The theory is then rewritten in a way that facilitates comparison with the 4-dimensional effective action for BIG, to be derived in the next section. In fact, the construction presented here for the FP theory enables us to make sense of the BIG effective action.

\subsection{Fierz-Pauli massive gravity in gauge invariant variables}

In standard 4-dimensional Einstein-Hilbert gravity, metric fluctuations $h_{\mu \nu}$ in a flat background can be made massive by introducing mass parameters, say, $a$ and $b$. The combination $a+b=0$ results in the ghost free Fierz-Pauli massive gravity theory [29, 30]. Since we are interested in the origin of ghost in brane induced gravity, we keep $a$ and $b$ unconstrained and use the term "Fierz-Pauli theory" in this generalized sense.

The gauge invariance of linearized gravity, broken by the mass terms, is restored by the Stückelberg technique. This amounts to performing a gauge transformation and retaining the gauge parameters as new fields. The resulting action is,

$$
S_{F P}=S_{E H}[h]-\frac{B}{4} \int d^{4} x\left[a\left(h_{\mu \nu}+2 \partial_{(\mu} \bar{f}_{\nu)}\right)^{2}+b\left(h_{\mu}^{\mu}+2 \partial_{\mu} \bar{f}^{\mu}\right)^{2}\right] .
$$

$S_{E H}[h]$ is read off from (2.6) for $d=4$. The $\bar{f}_{\mu}$ are Stückelberg fields transforming as $\delta \bar{f}_{\mu}=$ $\lambda^{\mu}$. Combined with $\delta h_{\mu \nu}=-2 \partial_{(\mu} \lambda_{\nu)}$, it keeps $S_{F P}$ invariant. The $\bar{f}_{\mu}$ can be interpreted as the Goldstone fields corresponding to the broken symmetry [51]. This conveys a sense of graviton masses arising due to spontaneous symmetry breaking, even in the absence of a more detailed fundamental mechanism. 
The theory can be rewritten in terms of $h^{\perp}$ and $s$ of (2.8) and the new gauge invariant variables,

$$
\bar{F}_{\mu}=\bar{f}_{\mu}+a_{\mu}+\frac{1}{2} \partial_{\mu} \phi .
$$

Note that these are the same as the $F_{\mu}$ in (2.17) once we identify the Stückelberg field as $\bar{f}_{\mu}=f_{\mu}+\left\langle A_{\mu}\right\rangle+\frac{1}{2} \partial_{\mu}(\langle\Phi\rangle)$, hence the similar notation. The Fierz-Pauli action (6.1) in terms of the gauge invariant variables becomes,

$$
\begin{gathered}
S_{F P}=-\frac{B}{4} \int d^{4} x\left[h^{\perp \mu \nu}(-\square+a) h_{\mu \nu}^{\perp}+s\left(\frac{3}{8} \square+\frac{a}{4}+b\right) s+4\left(\frac{a}{4}+b\right) \partial \cdot \bar{F} s\right. \\
\left.\left.+a\left(\partial_{\mu} \bar{F}_{\nu}-\partial_{\nu} \bar{F}_{\mu}\right)^{2}+4(a+b)(\partial \cdot \bar{F})^{2}\right)\right] .
\end{gathered}
$$

The equation of motion for $\bar{F}_{\mu}$,

$$
a \square \bar{F}_{\mu}+(a+2 b) \partial_{\mu}(\partial \cdot \bar{F})+\left(\frac{a}{4}+b\right) \partial_{\mu} s=0,
$$

has the purely longitudinal solution,

$$
\bar{F}_{\mu}=-\frac{\frac{a}{4}+b}{2(a+b)} \square^{-1} \partial_{\mu} s .
$$

Then, the $h_{\mu \nu}$ equation (obtained after expressing variations $\delta h^{\perp}$ and $\delta s$ in terms of $\delta h$ using the projection operators in Appendix A),

$$
(-\square+a) h_{\mu \nu}^{\perp}+\frac{4}{3}\left(\eta_{\mu \nu}-\frac{\partial_{\mu} \partial_{\nu}}{\square}\right)\left(\left(\frac{3}{8} \square+\frac{a}{4}+b\right) s+2\left(\frac{a}{4}+b\right) \partial \cdot \bar{F}\right)=T_{\mu \nu} / B,
$$

has the massive graviton solutions,

$$
\widetilde{h}_{\mu \nu}^{\perp}=\frac{1}{B} \frac{1}{k^{2}+a} \widetilde{T}_{\mu \nu}^{\perp}, \quad \widetilde{s}=-\frac{2}{3 B} \frac{\widetilde{T}}{k^{2}-\frac{1}{2} \frac{a}{a+b}(a+4 b)},
$$

corresponding to masses $m_{h^{\perp}}^{2}=a$ and $m_{s}^{2}=-\frac{1}{2} \frac{a}{a+b}(a+4 b)$. The full metric is obviously,

$$
h_{\mu \nu}=h_{\mu \nu}^{\perp}+\frac{1}{4} \eta_{\mu \nu} s+\partial_{\mu} \bar{F}_{\nu}+\partial_{\nu} \bar{F}_{\mu},
$$

with the gauge choice $\bar{f}_{\mu}=0$. In the following paragraphs, we comment on the ghost free theory before returning to the general case in the next subsection.

The massive FP gravity theory is, in general, not ghost free: A standard analysis of the gauge invariant amplitude (3.17) for interaction between sources $T_{\mu \nu}$ and $T_{\mu \nu}^{\prime}$ shows that for $a \neq 0$, the $s$ field is always a ghost ${ }^{15}$, as shown, for example, in [29, 31, 44] or

\footnotetext{
${ }^{15}$ The presence of the ghost for $a+b \neq 0$ can already be understood at the level of action $(6.3)$ in terms of the Stückelberg fields: The vector field $\bar{F}_{\mu}=F_{\mu}^{\perp}+\partial_{\mu} \phi_{F}$ has an unhealthy kinetic term because $(a+b)\left(\partial_{\mu} \bar{F}^{\mu}\right)^{2}$ leads to a 4-derivative term for the longitudinal mode $\phi_{F}$, resulting in ghost non-decoupling. For $a+b=0$, the 4 -derivative term is eliminated. This provides an intuitive way of detecting the massive gravity ghost.
} 
more explicitly in [45]. The only way to get rid of the ghost is to set $a+b=0$ which gives $m_{s}=\infty$ and $s=0$. The outcome is the ghost-free Fierz-Pauli massive gravity with ${ }^{16}$,

$$
\widetilde{h}_{\mu \nu}=\frac{1}{B}\left(\frac{1}{k^{2}+a} \widetilde{T}_{\mu \nu}^{\perp}+\frac{1}{3} \frac{k_{\mu} k_{\nu}}{a k^{2}} T\right), \quad(a+b=0) .
$$

An alternative solution to the ghost problem is to regard gravity as an effective theory and give the ghost a mass above the scale of validity of the effective theory by setting $a+b=\delta$ where $\delta$ is positive and very small. Then the inaccessible ghost pole becomes physically irrelevant [55].

Let us discuss an issue raised in section 4.4. While the ghost free theory has $s=0$ for a generic source, there is a caveat when $T_{\mu \nu}$ corresponds to a cosmological constant: When $a+b=0$, (6.4) implies $\square_{4} s=0$ and its solution $s=c_{1}+c_{2} s^{\text {harmonic }}$ replaces the expression for $s$ in terms of $\partial \cdot F$ obtained from (6.5). In general $c_{1,2}=0$ by boundary conditions, but for a cosmological constant source, the $h_{\mu \nu}$ equation gives $s=c_{1}=-4 \Lambda / 3 a B$. In section 4.4 , this behaviour was contrasted to that of brane induced gravity. For generic $a$ and $b$ the two theories respond to the cosmological constant in the same way.

\subsection{A 1-parameter family of actions for FP gravity}

From the outset it was clear that massive gravitons and ghosts were common features in both Fierz-Pauli theory (for $a+b \neq 0$ ) and BIG (for $n>2$ ) at the linearized level. Since FP gravity is simpler, it is natural to use it as a prototype to model some aspects of BIG, as in [38, 55]. But how much can the similarities be stretched? To quantify this, below we rewrite the Fierz-Pauli action in a way that helps us compare with, and make sense of, the BIG 4-dimensional effective action, to be derived in the next section.

As far as classical solutions are concerned, the action (6.3) is not unique. To find other equivalent actions, use the relation between $s$ and $\partial \cdot \bar{F}$ obtained from (6.5) to convert a part of the $s^{2}$ term into $(\partial \cdot \bar{F})^{2}$ and $s \partial \cdot \bar{F}$ terms. This gives,

$$
\begin{aligned}
S_{F P}= & -\frac{B}{4} \int d^{4} x\left[h^{\perp \mu \nu}(-\square+a) h_{\mu \nu}^{\perp}+\frac{3}{8} s \square s+\left(\frac{a}{4}+b+\bar{w}_{1}\right) s s\right. \\
& \left.\left.+4\left(\frac{a}{4}+b+\bar{w}_{2}\right) \partial \cdot \bar{F} s+a\left(\partial_{\mu} \bar{F}_{\nu}-\partial_{\nu} \bar{F}_{\mu}\right)^{2}+4\left(a+b+\bar{w}_{3}\right)\left(\partial_{\mu} \bar{F}^{\mu}\right)^{2}\right)\right],
\end{aligned}
$$

where, $\bar{w}_{1} s^{2}+4 \bar{w}_{2} \partial \cdot \bar{F} s+4 \bar{w}_{3}(\partial \cdot \bar{F})^{2}=0$ should hold on the classical solution. The $\bar{w}$ 's are further constrained by requiring that the above action leads to the correct equations of motion for $\bar{F}_{\mu}$ and $h_{\mu \nu}$. The outcome is,

$$
\bar{w}_{3}=\frac{a+b}{a / 4+b} \bar{w}_{2}, \quad \bar{w}_{2}=\frac{a+b}{a / 4+b} \bar{w}_{1} .
$$

\footnotetext{
${ }^{16}$ The origin of vDVZ [31, 32, 33] discontinuity, that arises in the massless limit of ghost-free FP theory, is manifest in this formulation: Sending $m_{h^{\perp}} \rightarrow 0$, keeping $m_{s}=\infty$ fixed, does not recover the standard massless solutions 3.18 as $s$ remains zero and the theory does not couple to $T_{\mu}^{\mu}$. The difference between the two massless theories does not affect light for which $T_{\mu}^{\mu}=0$. But matter is affected differently and the definitions of the Newton constant in the two theories differ by a factor of $3 / 4$. This shows up in comparing the bending of light in the two cases. The discontinuity is avoided in the non-linear theory through the Vainshtein effect [39, 40].
} 
Thus we get a 1-parameter family of actions, in terms of gauge invariant variables, producing the same equation of motion as the Fierz-Pauli theory.

A point to note is that, except for $\bar{w}_{1}=0$, these equivalent actions will look non-local when expressed in terms of $h_{\mu \nu}$, with $\square^{-1}$-type non-localities arising from the projection operators (A.1) in Appendix A.

The particular choice $\bar{w}_{3}=-(a+b)$ (implying $\bar{w}_{2}=-(a / 4+b)$ ) decouples gravity from $\bar{F}_{\mu}$ which become a free Abelian gauge field. The resulting action involves only $h^{\perp}$ and $s$ (corresponding to integrating out $\bar{F}_{\mu}$ ),

$$
S_{F P}=-\frac{B}{4} \int d^{4} x\left[h^{\perp \mu \nu}(-\square+a) h_{\mu \nu}^{\perp}+\frac{3}{8} s\left(\square+\frac{a(a+4 b)}{2(a+b)}\right) s\right] .
$$

Note that, although the solutions (6.7) can be readily obtained from here, this choice of $\bar{w}_{3}$ is a singular limit of $(6.8)$ in the sense that $\partial \cdot F$ becomes undetermined ${ }^{17}$.

When re-expressed in terms of $h_{\mu \nu}$, using projection operators of Appendix A, this action remains manifestly gauge invariant, with no dependence on Stückelberg fields and no sign of symmetry breaking. But, as a trade off, it will contain $\square^{-1}$-type non-localities arising from projection operators. In this example, we know that these non-localities arise as a result of integrating out the $\bar{F}_{\mu}$ and indicate the existence of a local formulation ( 6.1 or (6.3)) in which Stückelberg fields are needed to restore gauge invariance. The two cases are connected by the interpolating action (6.8). This construction is useful for obtaining the 4-d effective action in brane induced gravity where the above steps are traversed in the reverse order.

\section{4-d Effective Action in BIG and Broken Gauge Invariance}

In order to better understand the 4-dimensional structure of BIG, we need an effective 4-dimensional description of it. In this section we work out such an effective action by integrating out parts of the fields with support away form the brane as well as the extra components of the metric. Then we construct an action containing the Stückelberg-like fields $F_{\mu}$, based on our understanding of massive gravity in the previous section.

This has implications for the origin of ghost and for the realization of the brane general covariance as a spontaneously broken gauge symmetry: The $F_{\mu}$ or $f_{\mu}$ (2.17) of BIG are closely related to Stückelberg fields $\bar{f}_{\mu}$ in Fierz-Pauli theory (more precisely, $\bar{f}_{\mu} \sim f_{\mu}+$ $\left.\left\langle A_{\mu}\right\rangle+\frac{1}{2} \partial_{\mu}(\langle\Phi\rangle)\right)$. Now, $\bar{f}_{\mu}$ restore gauge invariances broken by the FP mass terms and, hence, can be interpreted as the Goldstone fields associated with the broken symmetry [51. The appearance of their analogues $f_{\mu}$ in BIG is thus a manifestation of spontaneous breakdown of 4-dimensional general covariance by the bulk-brane setup. In this sense, BIG provides a realization of the gravitational Higgs mechanism.

\subsection{4-dimensional effective action in Brane Induced Gravity}

In this subsection we derive a 4-dimensional effective action for BIG by integrating out all bulk related modes in the $A$ and $B$ terms of (2.1). The bulk field $H_{M N}\left(x_{\|}, x_{\perp}-y_{0}\right)$ has

\footnotetext{
${ }^{17}$ This is obvious from the $F_{\mu}$ equation of motion for 6.8 which gives, $2\left(a+b+\bar{w}_{3}\right) \partial \cdot F=-\left(a / 4+b+\bar{w}_{2}\right) s$
} 
a part $\left\langle H^{\perp}\right\rangle_{M N}\left(x_{\|}\right)$, given by $(2.10)$, that has support only on the brane. The remaining part $\Delta H_{M N}\left(x_{\|}, x_{\perp}-y_{0}\right)$ has no support on the brane, i.e., $\left\langle\Delta H_{M N}\right\rangle=0$, and is given by the decomposition,

$$
H_{M N}=\Delta H_{M N}+\frac{P\left(x_{\perp}-y_{0}\right)}{Z}\langle H\rangle_{M N},
$$

where, $Z=\int d^{n} x_{\perp} P^{2}=(2 \pi)^{n} \int d^{n} q \widetilde{P}^{2}$. In terms of momentum space variables,

$$
\widetilde{H}_{M N}(k, q)=\Delta \widetilde{H}_{M N}(k, q)+\frac{\widetilde{P}(q)}{Z}\langle\widetilde{H}\rangle_{M N}(k) .
$$

To determine the effective action on the brane, we eliminate, first, $\Delta H_{M N}$ by using its equation of motion and then solve for $\langle H\rangle_{i j}$ and $\langle H\rangle_{\mu j}$ using the corresponding equations. This leaves us with an effective action for $\langle H\rangle_{\mu \nu}$ or, equivalently, an action for the 4 dimensional brane variables $h_{\mu \nu}^{\perp}, s$, and $F^{\mu}$.

To integrate out $\Delta H_{M N}$, start with the bulk action (2.6), with $H_{M N}$ given by the above decomposition, and a Lagrange multiplier term implementing the condition $\langle\Delta H\rangle_{M N}=0$,

$$
S_{E H}^{b u u l k}+\int d^{d} x\left(P \Delta H_{M N}\right) L^{M N}\left(x_{\|}\right) .
$$

To insure gauge invariance, $L^{M N}$ must satisfy $\partial_{M}\left(P L^{M N}\right)=0$. The momentum space equation of motion for the variation $\delta H_{M N}=\delta\left(\Delta H_{M N}\right)$ (with no brane support) is,

$$
\frac{A}{2}\left[p^{2} \widetilde{H}^{\perp M N}+\frac{d-2}{d}\left(p^{M} p^{N}-\eta^{M N} p^{2}\right) \widetilde{S}\right]-\widetilde{L}^{M N}(k) \widetilde{P}(q)=0 .
$$

This can be easily solved for $\widetilde{S}$ and $\widetilde{H}^{\perp M N}$ in terms of $\widetilde{L}=\widetilde{L}_{M}^{M}$ and $\widetilde{L}^{M N}$. Blurring the solutions using (2.11) then determines $\widetilde{L}$ and $\widetilde{L}^{M N}$ in terms of $\langle\widetilde{S}\rangle$ and $\left\langle\widetilde{H}^{\perp}\right\rangle^{M N}$. Substituting back in the solutions for $\widetilde{S}$ and $\widetilde{H}^{\perp M N}$ gives (3.5) and,

$$
\widetilde{H}^{\perp M N}=\frac{\widetilde{P}}{k^{2}+q^{2}} \frac{1}{\langle\widetilde{G}\rangle}\left[-\left\langle\widetilde{H}^{\perp}\right\rangle^{M N}+\frac{d-2}{d}\left(\frac{\langle\widetilde{g}\rangle^{M N}}{\langle\widetilde{G}\rangle}+\frac{p^{M} p^{N}}{k^{2}+q^{2}}\right)\langle\widetilde{S}\rangle\right],
$$

where we have introduced (as a generalization of (3.8))

$$
\langle\widetilde{g}\rangle^{M N}=(2 \pi)^{n} \int d^{n} q \frac{p^{M} p^{N} \widetilde{P}^{2}(q)}{\left(k^{2}+q^{2}\right)^{2}} .
$$

Substituting this back in $S_{E H}^{b u l k}$ gives a 4-dimensional action for $\left\langle\widetilde{H}^{\perp}\right\rangle^{M N}$ and $\langle\widetilde{S}\rangle$ (the contribution of the Lagrange multiplier term vanishes),

$$
\begin{aligned}
S_{E H}^{\text {bulk,eff }}=(2 \pi)^{4} \frac{A}{4} \int d^{4} k \frac{1}{\langle\widetilde{G}\rangle}\left[\left\langle\widetilde{H}^{\perp}\right\rangle^{M N}\left\langle\widetilde{H}^{\perp}\right\rangle_{M N}^{*}\right. & -\frac{(d-2)(d-1)}{d^{2}}\langle\widetilde{S}\rangle\langle\widetilde{S}\rangle^{*} \\
& \left.-\frac{d-2}{d} \frac{\langle\widetilde{g}\rangle_{M N}}{\langle\widetilde{G}\rangle}\left\langle\widetilde{H}^{\perp}\right\rangle^{M N}\langle\widetilde{S}\rangle^{*}\right] .
\end{aligned}
$$

From this we can further eliminate $\left\langle\widetilde{H}^{\perp}\right\rangle_{i j}$ and $\left\langle\widetilde{H}^{\perp}\right\rangle_{i \mu}$ by expressing them in terms of $\langle\widetilde{S}\rangle$ using the solutions of the $(i, j)$ and $(\mu, j)$ components of (3.2) along with (3.5). Then, we 
express $\left\langle\widetilde{H}^{\perp}\right\rangle_{\mu \nu}$ and $\langle\widetilde{S}\rangle$ in terms of $\widetilde{h}_{\mu \nu}^{\perp}, \widetilde{s}$ and $\widetilde{F}_{\mu}$ using the surface equation $(2.16)$ and its consequences. Note that the equations used so far also determine $\widetilde{F}_{\mu}$ in terms of $\widetilde{s}(3.14)$, but leave $\widetilde{s}$ and $\widetilde{h}^{\perp}$ undetermined. Finally, adding $S_{E H}^{\text {brane }}[h]$ leads to the 4 -dimensional effective action,

$$
S_{\text {eff }}[h]=(2 \pi)^{4} \frac{B}{4} \int d^{4} k\left[\left(-k^{2}+\frac{A}{B}\langle\widetilde{G}\rangle^{-1}\right) \widetilde{h}^{\perp \mu \nu} \widetilde{h}_{\mu \nu}^{\perp *}+\frac{3}{8}\left(k^{2}+\frac{A}{2 B} \frac{d-2}{d-5}\langle\widetilde{G}\rangle^{-1}\right) \widetilde{s} \widetilde{s}^{*}\right] .
$$

This gives the correct equation of motion with the correct solutions (3.15), (3.16). However, it is not yet the final form of the effective action, as explained below.

The above action is the analogue of the Fierz-Pauli action in the form (6.10) which, as argued there, is not its most natural (i.e., most local) form. In fact, the $h^{\perp}$-terms in the two actions can be mapped by replacing the FP mass parameter $a$ as,

$$
a \rightarrow \widetilde{a}(k)=-\frac{A}{B}\langle\widetilde{G}\rangle(k) .
$$

Naively, the $s$-terms too can be mapped by replacing the FP $b$ parameter by $\widetilde{a}(k) /(d-6)$. But that is not the correct comparison since it does not map the $\bar{F}_{\mu}$ solution of FP theory (6.5) to the $F_{\mu}$ solution of BIG (3.14) (simply because the quantity $U$ does not appear in the map). In spite of this, the similarity between the BIG action (7.8) and the FP action in the form $(6.10)$, teaches us the following:

Recall that in the FP case, (6.10) was a singular limit of a 1-parameter family of actions (6.8), a generic member of which contained the FP Stückelberg fields $\bar{F}_{\mu}$. The $\bar{w}_{1}=0$ member of this family was the original FP action (6.1) which was local when written in terms of $h_{\mu \nu}$ and $\bar{f}_{\mu}$. However, the specific $\bar{w}_{1}$ choice that led to (6.10) decoupled $\bar{F}_{\mu}$, so the corresponding solution could not be reproduced. Also this action had $1 / k^{2}$-type non-localities when expressed in terms of $h_{\mu \nu}$.

To compare, this also is the structure of the BIG effective action (7.8). It too does not contain the worldvolume fields $F_{\mu}$ and, when expressed in terms of $h_{\mu \nu}$, will contains $1 / k^{2}$-type non-localities arising from the projection operators (A.1) ${ }^{18}$. We take this as an indication that (7.8), too, is the singular limit of a 1-parameter family of actions that generically contain the $F_{\mu}$, beside the metric.

The fact that the replacements for $a$ and $b$ described above cannot map the $\bar{F}_{\mu}$ solution to the $F_{\mu}$ solution means that the 1-parameter family to which the BIG effective action (7.8) belongs cannot be obtained from the corresponding construct (6.8) in FP theory. In this sense, the two 1-parameter families are not equivalent. The explicit construction of the BIG 1-parameter family is performed below and is then compared to the FP theory for a different choice of $b$.

\subsection{A 1-parameter family of 4-d BIG effective actions}

To reinstate the $F_{\mu}$ into the BIG effective action, let's get back to equation (7.7). We again eliminate the bulk tensors in favour of $h^{\perp}, s$ and $F_{\mu}$, but now retain $F_{\mu}$. Since the relation

\footnotetext{
${ }^{18}$ Of course, the action $(7.8)$ also contains non-localities through the $k$-dependence of $k^{2}\langle\widetilde{G}\rangle$. But these arise as a result of integrating out bulk modes and are not related to Stückelberg fields.
} 
between $s$ and $F_{\mu}$ is already implicit in the equations employed, the outcome is not unique and depends on the steps followed. Depending on the details, the resulting action may not even lead to the correct equations of motion for $h_{\mu \nu}$ and $F_{\mu}$. But this can be corrected. Indeed, a candidate action that one directly obtains in this way is ${ }^{19}$,

$$
\begin{aligned}
S_{E H}^{\text {bulk,eff }}= & (2 \pi)^{4} \frac{A}{4} \int d^{4} k\left[\frac{1}{\langle\widetilde{G}\rangle} \widetilde{h}^{\perp \mu \nu} \widetilde{h}_{\mu \nu}^{\perp *}+\frac{U_{1}}{\langle\widetilde{G}\rangle} \widetilde{s s}^{*}\right. \\
& \left.+\frac{2}{\langle\widetilde{G}\rangle}\left(k^{2} \widetilde{F}^{2}+(k \cdot \widetilde{F})^{2}\right)+i \frac{U_{2}}{\langle\widetilde{G}\rangle}\left(k \cdot \widetilde{F}^{*} \widetilde{s}-k \cdot \widetilde{F} \widetilde{s}^{*}\right)\right],
\end{aligned}
$$

with,

$$
\begin{aligned}
& U_{1}=\frac{1}{16} \frac{(d-2)^{2}}{(d-1)^{2}(d-5)^{2}}\left[2(d-4)(2 d-5)-9 \frac{d-1}{d-2}-3(d-7) \frac{\langle\widetilde{g}\rangle}{\langle\widetilde{G}\rangle}\right], \\
& U_{2}=\frac{1}{4} \frac{(d-2)}{(d-1)(d-5)}\left[2(d-4)-3-3 \frac{\langle\widetilde{g}\rangle}{\langle\widetilde{G}\rangle}\right] .
\end{aligned}
$$

This does not reproduce the correct equations of motion (after adding $S_{E H}^{b r a n e}[h]$ ), indicating that the ambiguity between $s$ and $\partial \cdot F$ terms has not yet been properly resolved. To cure this, one can use the relation between $s$ and $\partial \cdot F$ in (3.14) to write an appropriate part of the $\widetilde{s}^{*} \widetilde{s}$ term in terms of $k \cdot \widetilde{F}$. As a shortcut, a simple inspection shows that the correct solution $\widetilde{F}_{\mu}=-i U\left(k_{\mu} / 4 k^{2}\right) \widetilde{s}$ can be obtained if $U_{2}$ is, somehow, replaced by $U$ given by (3.14). Hence, the trick is to set $U_{2}=U+\delta U_{2}$ and then convert the coefficient of the $\delta U_{2}$ piece to $\widetilde{s} \widetilde{s}^{*}$ form using (3.14). This leads to an effective action containing $F_{\mu}$ that reproduces the correct equations of motion,

$$
\begin{gathered}
S_{e f f}[h, F]=S_{E H}^{\text {brane }}[h]+(2 \pi)^{4} \frac{A}{4} \int d^{4} k\left[\frac{1}{\langle\widetilde{G}\rangle} \widetilde{h}^{\perp \mu \nu} \widetilde{h}_{\mu \nu}^{\perp *}+\frac{1}{4\langle\widetilde{G}\rangle}\left(\frac{3}{4} \frac{d-2}{d-5}+U^{2}\right) \widetilde{s}^{*}\right. \\
\left.+\frac{2}{\langle\widetilde{G}\rangle}\left(k^{2} \widetilde{F}^{2}+(k \cdot \widetilde{F})^{2}\right)+i \frac{U}{\langle\widetilde{G}\rangle}\left(k \cdot \widetilde{F}^{*} \widetilde{s}-k \cdot \widetilde{F} \widetilde{s}^{*}\right)\right] .
\end{gathered}
$$

But, as experience with FP theory shows, this action may not be unique in this respect. Consider converting a part of the $s s^{*}$ term into $(\partial F)^{2}$ and $\partial F s$ terms, thereby, shifting the corresponding coefficients by $\omega_{1}, \omega_{2}$ and $\omega_{3}$ to (for convenience we use the notation (7.9)),

$$
\begin{array}{r}
S_{e f f}[h, F]=-(2 \pi)^{4} \frac{B}{4} \int d^{4} k\left[\left(k^{2}+\widetilde{a}\right) \widetilde{h}^{\perp \mu \nu} \widetilde{h}_{\mu \nu}^{\perp *}-\left(\frac{3}{8} k^{2}-\widetilde{a}\left(\frac{3}{16} \frac{d-2}{d-5}+\frac{U^{2}}{4}+w_{1}\right)\right) \widetilde{s s}^{*}\right. \\
\left.\quad+i\left(U+w_{2}\right) \widetilde{a}\left(k \cdot \widetilde{F}^{*} \widetilde{s}-k \cdot \widetilde{F} \widetilde{s}^{*}\right)+\widetilde{a}\left(k_{\mu} \widetilde{F}_{\nu}-k_{\nu} \widetilde{F}_{\mu}\right)^{2}+\widetilde{a}\left(4+w_{3}\right)(k \cdot \widetilde{F})^{2}\right] .(7 .
\end{array}
$$

Clearly, the modification is constrained by $w_{1} \widetilde{s}^{*}+i w_{2}\left(k \cdot \widetilde{F}^{*} \widetilde{s}-k \cdot \widetilde{F} \widetilde{s}^{*}\right)+w_{3}(k \cdot \widetilde{F})^{2}=0$. Then the equations of motion reproduce the correct solutions provided,

$$
w_{3}=\frac{4}{U} w_{2}, \quad w_{2}=\frac{4}{U} w_{1} .
$$

\footnotetext{
${ }^{19}$ We have rewritten $k^{\mu} k^{\nu}\left\langle\widetilde{H}^{\perp}\right\rangle_{\mu \nu}\langle\widetilde{S}\rangle$ using the surface equation 2.16 ). Instead, writing $k^{\mu} k^{\nu}\left\langle\widetilde{H}^{\perp}\right\rangle_{\mu \nu}=$ $\left\langle q_{i} q_{j} \widetilde{H}^{\perp i j}\right\rangle$ and using the second equation in (3.10) would have given a different result.
} 
Hence, we have a 1-parameter family of correct BIG effective actions.

The $F_{\mu}$ equation of motion,

$$
2 k^{2} \widetilde{F}_{\mu}+\left(2+w_{3}\right) k_{\mu}(k \cdot \widetilde{F})+i\left(U+w_{2}\right) k_{\mu} \widetilde{s}=0,
$$

gives the correct $F_{\mu}$ solution (3.14), except for $w_{2}=-U$ (implying $w_{3}=-4$ ) which leads again to the action (7.8). For this special value, $\partial^{\mu}\left(\partial_{\mu} F_{\nu}-\partial_{\nu} F_{\mu}\right)=0$ and $F_{\mu}$ is pure gauge, but otherwise undetermined. Hence, as expected, (7.8) arises as the singular limit of the family (7.13). The the complete effective action, capable of determining all worldvolume fields $h^{\perp}, s$ and $F$, should be a non-singular element of this family.

The above discussion also highlights a structural similarity between the BIG and FP actions in the forms (7.13) and (6.8) which are inequivalent otherwise: The specific choices of the parameters $w_{i}$ and $\bar{w}_{i}$ that decouple $F_{\mu}$ and $\bar{F}_{\mu}$, respectively, from gravity, also convert the $F$ 's into free Maxwell fields. This, in particular, decouples the longitudinal mode of the vector field which is ghost-like, thereby, transferring the ghost entirely to the gravity sector. In this respect, the ghosts in BIG (for $n>1$ ) and in FP theory (for $a+b \neq 0$ ) have similar origins, both being related to the survival of the gauge dependent components of the brane metric (contained in $F_{\mu}$ ).

\subsection{Spontaneous breaking of 4-d gauge invariance}

The discussion in the previous section shows that the correct BIG effective action is a nonsingular member of the family (7.13). The presence of $F_{\mu}$ in this action has the outcome that gauge dependent components of the metric, $a_{\mu}$ and $\phi$ (2.8), do not drop out of the action and gauge invariance is maintained only through the compensating transformations of $f_{\mu}$. Thus, the 4-dimensional gauge invariance of the theory, i.e., symmetry under $\delta h_{\mu \nu}=-2 \partial_{(\mu} \lambda_{\nu)}$, which is manifest in the starting BIG action, is broken spontaneously in the effective 4dimensional theory, as signaled by the need for compensating transformations.

This effect can be traced to the fact that the process of integrating out non-brane components of graviton, by partially solving the equations of motion, requires eliminating gauge degrees of freedom through either explicit gauge fixing, or the use of gauge invariant variables. In our approach, this is technically related to the necessity of using (2.16) in relating gauge invariant bulk and brane variables. In other approaches, the same issue will arise though it may be obscured by an explicit gauge fixing.

To make the spontaneously broken nature of the symmetry very explicit, we can write the effective action in terms of the gauge non-invariant variables $h_{\mu \nu}$ and $f_{\mu}$. A familiar form is obtained by matching some terms of the BIG effective action (7.13) (by choosing an appropriate $w_{1}$ ) to the starting form of the FP action (6.3). For example, we can map the coefficients of the $s^{2}$ and $(\partial F) s$ terms to FP theory by choosing

$$
w_{1}=-\frac{3}{16} \frac{d-2}{d-5}\left(\frac{U}{U+2}\right)-\frac{U^{2}}{4} .
$$

Then, in terms of

$$
\widetilde{a}(k)=-\frac{A}{B}\langle\widetilde{G}\rangle(k), \quad \widetilde{b}(k)=\frac{\widetilde{a}}{4}\left(\frac{3}{2} \frac{d-2}{d-5} \frac{1}{U+2}-1\right),
$$


the BIG effective action takes a form very similar to the FP action, differing from it only in the $(\partial F)^{2}$ term. Finally, in terms $h_{\mu \nu}$ and $f_{\mu}$, it becomes,

$$
\begin{aligned}
S_{e f f}[h, f]=S_{E H}[h]-\frac{B}{4} \int d^{4} k\left[\widetilde{a}(k)\left|\widetilde{h}_{\mu \nu}+2 i k_{(\mu} \widetilde{f}_{\nu)}\right|^{2}+\widetilde{b}(k)\left|\widetilde{h}_{\mu}^{\mu}+2 i k_{\mu} \widetilde{f}^{\mu}\right|^{2}\right. \\
\left.-2 \widetilde{b}(k) \frac{2 U+1}{U}\left|k \cdot \widetilde{f}+\frac{1}{6} \widetilde{h}-\frac{2}{3} \frac{k^{\mu} k^{\nu}}{k^{2}} h_{\mu \nu}\right|^{2}\right] .
\end{aligned}
$$

The first line is an exact map from the FP theory. The second line shows the structural difference between massive FP gravity and the BIG effective action for $n>1$.

Finally, one may comment on the similarity of brane induced gravity with the gravitational Higgs mechanism of ([35, 36]). In these works, in order to break general covariance, one introduces 4 scalars fields $\phi^{\mu}(\sigma)=\sigma^{\mu}+\delta \phi^{\mu}$, with a Minkowski metric on the field space ( $\sigma^{\mu}$ denote 4 dimensional spacetime coordinates). These fields are very similar to our brane embedding coordinates, $x^{\mu}(\sigma)=\sigma^{\mu}+f^{\mu}$ which naturally come with a Minkowski metric (the transverse fluctuations $y^{i}$ are not relevant). In this sense, BIG provides a natural realization of Higgs mechanism in gravity.

Note that the presence of extra dimensions is crucial for this similarity to hold. The reason is that, as discussed earlier, the $f^{\mu}$ are non-trivial only for $n>0$. It is interesting to explore the connection between these two theories further.

\section{Conclusions}

Our results are summarized and discussed in detail in section 1. Here we summarize the main conclusions. The existing classical solutions for bulk gravity sourced by a 3brane tension are not adequate for studying metric fluctuations on the brane. Hence we use the flat background approximation but argue that the results also shed light on the curved background analysis. In the flat approximation, brane induced gravity filters out a cosmological constant using the mass of the unhealthy scalar mode. This mass is partly an artifact of the flat approximation and there is no real tachyon instability (in the sense of an unbounded Hamiltonian) associated with it.

The presence of ghost in BIG is related to the phenomenologically interesting values of parameters in the theory, corresponding to a very low graviton mass and effectively 4-dimensional gravity on the brane (for example, there is no problem with the natural parameter values in the string theory D-brane setups). For these parameter values avoiding the ghost is difficult even in a curved background sourced by $\Lambda$ since our analysis can already probe such backgrounds for small values of $\Lambda$ and the problem is very similar to ghosts in ordinary massive gravity. If this turns out to be the case, then BIG cannot be regarded as a viable solution to the cosmological constant problem.

The only healthy exception would be a background that absorbs the cosmological constant by curving directions transverse to the brane while leading to massless gravity on the brane $\left(\Sigma_{\text {curved }}(0)=0\right)$, if that is possible at all. This would avoid the ghost issue and lead to a theory with filtered out $\Lambda$ and modified massless gravity on the brane. The 
modifications to gravity can be computed using the methods of section 3.3 and will have observable consequences. However, verifying this possibility requires constructing brane classical solutions that satisfy the equations of motion with the correct source terms. As indicated earlier, finding such solutions is a generic problem in brane physics. At the moment, we cannot say more on this possibility (however, see see footnote 3 ).

Another conclusion is that, using gauge invariant variables, it is possible to keep track of symmetries and explicitly show that, in the effective 4-dimensional gravity theory, gauge dependent components of the metric do not decouple. This indicates a spontaneous breaking of 4-dimensional general covariance as a consequence of the bulk-brane setup. This setup generates the extra gauge invariant modes that contain the Stückelberg fields associated with the spontaneous breakdown of symmetry. It provides a realization of the gravitational Higgs mechanism.

\section{Acknowledgments}

We would like to thank I. Antoniadis, M. Baumgartl, F. Berkhahn, C. Burgess, M. Berg, C. Deffayet, J. Enander, C. de Rahm, G. Gabadadze, M. R. Garousi, C. Germani, A. Ghodsi, H.T. Hansson, K. Hinterbichler, J. Khoury, E. Kiritsis, P. Moyasseri, S. Ramgoolam, R. Rosen, B. Sundborg, A. Tolley, for useful discussions and comments.

\section{A. Projection operators for gauge invariant variables}

For a symmetric tensor $H_{M N}$, its transverse part $H_{M N}^{\prime}$ satisfying $\partial^{M} H_{M N}^{\prime}=0$ is given by

$$
H_{M N}^{\prime}=H_{M N}-\square_{d}^{-1}\left(\partial_{M} \partial^{L} H_{L N}+\partial_{N} \partial^{L} H_{M L}-\square_{d}^{-1} \partial_{M} \partial_{N} \partial^{L} \partial^{K} H_{L K}\right)
$$

Then, the components in the decomposition (2.5) are given by (with $H=H_{L}^{L}$ ),

$$
\begin{array}{ll}
H_{M N}^{\perp}=H_{M N}^{\prime}-\frac{1}{d-1}\left(\eta_{M N}-\frac{\partial_{M} \partial_{N}}{\square_{d}}\right) H^{\prime}, & S=\frac{d}{d-1}\left(H-\frac{\partial^{L} \partial^{K} H_{L K}}{\square_{d}}\right), \\
A_{N}=\frac{1}{\square_{d}}\left(\partial^{L} H_{L N}-\partial_{N} \frac{\partial^{L} \partial^{K} H_{L K}}{\square_{d}}\right), & \Phi=\frac{d}{d-1}\left(\frac{\partial^{L} \partial^{K} H_{L K}}{\square_{d} \square_{d}}\right)-\frac{1}{d-1} \frac{H}{\square_{d}}
\end{array}
$$

The gauge transformation $\delta H_{M N}=-2 \partial_{(M} \xi_{N)}$ results from the variation of $A_{M}+\frac{1}{2} \partial_{M} \Phi$. $H_{M N}^{\perp}$ and $S$ are invariant except under a restricted class of transformation with $\square_{d} \xi^{M}=0$. These become relevant when $\square_{d} H_{M N}=0$ which is not the case in brane induced gravity.

The energy-momentum tensor $T_{M N}$ can be decomposed in a similar way,

$$
T_{M N}=T_{M N}^{\perp}+\partial_{M} T_{N}+\partial_{N} T_{M}+\partial_{M} \partial_{N} T_{\phi}+\frac{1}{d} \eta_{M N} T_{s}
$$

The components in this expansion are given by expressions analogous to those for $H_{M N}$ in terms of $T_{M N}$ and a $T_{M N}^{\prime}$.

Generically, $\square_{d} T_{M N} \neq 0$. Then $\partial^{M} T_{M N}=0$ implies $T_{M N}^{\prime}=T_{M N}$ and other simplifications. However, if $\square_{d} T_{M N}=0$, then $\square_{d}^{-1} \partial^{L} \partial^{K} T_{L K} \neq 0$ and one should not use the simplified equations. An example is the cosmological constant source $T_{M N}^{\Lambda}=\eta_{M N} \Lambda$. Then, the simplified equations do not hold, while using the complete projection operators one gets, $T_{M N}^{\Lambda \perp}=T_{M}^{\Lambda}=T_{\phi}^{\Lambda}=0$ and $T_{s}^{\Lambda}=d \Lambda$. 


\section{References}

[1] G. R. Dvali, G. Gabadadze and M. Porrati, Phys. Lett. B 485 (2000) 208 [arXiv:hep-th/0005016].

[2] G. R. Dvali and G. Gabadadze, Phys. Rev. D 63, 065007 (2001) [arXiv:hep-th/0008054].

[3] G. Gabadadze, arXiv:hep-th/0408118.

[4] S. Weinberg, Rev. Mod. Phys. 61 (1989) 1.

[5] G. Dvali, G. Gabadadze and M. Shifman, Phys. Rev. D 67, 044020 (2003) [arXiv:hep-th/0202174].

[6] G. Dvali, G. Gabadadze and M. Shifman, arXiv:hep-th/0208096.

[7] R. Bousso and J. Polchinski, JHEP 0006 (2000) 006 [arXiv:hep-th/0004134].

[8] D. A. Demir, Found. Phys. 39 (2009) 1407 [arXiv:0910.2730 [hep-th]].

[9] J. Polchinski, arXiv:hep-th/0603249.

[10] S. Nobbenhuis, Found. Phys. 36 (2006) 613 [arXiv:gr-qc/0411093].

[11] S. Nobbenhuis, arXiv:gr-qc/0609011.

[12] C. de Rham, S. Hofmann, J. Khoury and A. J. Tolley, JCAP 0802 (2008) 011 [arXiv:0712.2821 [hep-th]].

[13] C. de Rham, J. Khoury and A. J. Tolley, Phys. Rev. D 81 (2010) 124027 [arXiv:1002.1075 [hep-th]].

[14] R. Gregory, Nucl. Phys. B 467 (1996) 159 [arXiv:hep-th/9510202].

[15] C. Charmousis, R. Emparan and R. Gregory, JHEP 0105 (2001) 026 [arXiv:hep-th/0101198].

[16] V. A. Rubakov and M. E. Shaposhnikov, Phys. Lett. B 125 (1983) 139.

[17] G. Dvali, G. Gabadadze, X. r. Hou and E. Sefusatti, Phys. Rev. D 67, 044019 (2003) [arXiv:hep-th/0111266].

[18] S. Corley, D. A. Lowe and S. Ramgoolam, JHEP 0107 (2001) 030 [arXiv:hep-th/0106067].

[19] F. Ardalan, H. Arfaei, M. R. Garousi and A. Ghodsi, Int. J. Mod. Phys. A 18, 1051 (2003) [arXiv:hep-th/0204117].

[20] I. Antoniadis, R. Minasian and P. Vanhove, Nucl. Phys. B 648, 69 (2003) [arXiv:hep-th/0209030].

[21] E. Kohlprath and P. Vanhove, arXiv:hep-th/0409197.

[22] F. T. J. Epple, JHEP 0409 (2004) 021 [arXiv:hep-th/0408105].

[23] E. Kiritsis, N. Tetradis and T. N. Tomaras, JHEP 0108, 012 (2001) [arXiv:hep-th/0106050].

[24] O. Corradini, A. Iglesias, Z. Kakushadze and P. Langfelder, Phys. Lett. B 521 (2001) 96 [arXiv:hep-th/0108055].

[25] N. Kaloper and D. Kiley, JHEP 0705 (2007) 045 [arXiv:hep-th/0703190].

[26] N. Kaloper, Mod. Phys. Lett. A 23 (2008) 781 [arXiv:0711.3210 [hep-th]]. 
[27] G. Gabadadze and M. Shifman, Phys. Rev. D 69, 124032 (2004) [arXiv:hep-th/0312289].

[28] S. L. Dubovsky and V. A. Rubakov, Phys. Rev. D 67, 104014 (2003)

[arXiv:hep-th/0212222].

[29] M. Fierz, Helv. Phys. Acta 12 (1939) 3.

[30] M. Fierz and W. Pauli, Proc. Roy. Soc. Lond. A 173 (1939) 211.

[31] H. van Dam and M. J. G. Veltman, Nucl. Phys. B 22 (1970) 397.

[32] V. I. Zakharov, JETP Lett. 12 (1970) 312 [Pisma Zh. Eksp. Teor. Fiz. 12 (1970) 447].

[33] Y. Iwasaki, Phys. Rev. D 2 (1970) 2255.

[34] M. Kolanovic, M. Porrati and J. W. Rombouts, Phys. Rev. D 68 (2003) 064018 [arXiv:hep-th/0304148].

[35] G. 't Hooft, arXiv:0708.3184 [hep-th].

[36] Z. Kakushadze, Phys. Rev. D 77, 024001 (2008) [arXiv:0710.1061 [hep-th]].

[37] N. Arkani-Hamed, S. Dimopoulos, G. Dvali and G. Gabadadze, arXiv:hep-th/0209227.

[38] G. Dvali, S. Hofmann and J. Khoury, Phys. Rev. D 76 (2007) 084006 [arXiv:hep-th/0703027].

[39] A. I. Vainshtein, Phys. Lett. B 39 (1972) 393.

[40] C. Deffayet, G. R. Dvali, G. Gabadadze and A. I. Vainshtein, Phys. Rev. D 65 (2002) 044026 [arXiv:hep-th/0106001].

[41] S. Weinberg, "Gravitation and Cosmology: Principles and Applications of the General Theory of Relativity", J. Wiley and Sons (1972) 657 p.

[42] S. L. Adler, Rev. Mod. Phys. 54 (1982) 729 [Erratum-ibid. 55 (1983) 837].

[43] I. R. Klebanov and L. Thorlacius, Phys. Lett. B 371 (1996) 51 [arXiv:hep-th/9510200].

[44] P. Van Nieuwenhuizen, Nucl. Phys. B 60 (1973) 478.

[45] F. C. P. Nunes and G. O. Pires, Phys. Lett. B 301 (1993) 339.

[46] M. Abramowitz and I. A. Stegun, "Handbook of Mathematical Functions", Dover Publications (1965) $1046 p$

[47] M. E. Peskin and D. V. Schroeder, "An Introduction To Quantum Field Theory," Reading, USA: Addison-Wesley (1995) $842 p$

[48] L. S. Brown, "Quantum Field Theory," Cambridge, UK: Univ. Pr. (1992) $542 p$

[49] H. Gustafsson, U. Lindstrom, P. Saltsidis, B. Sundborg and R. van Unge, Nucl. Phys. B 440 (1995) 495 [arXiv:hep-th/9410143].

[50] P. L. Bozhilov, arXiv:hep-th/0011032.

[51] N. Arkani-Hamed, H. Georgi and M. D. Schwartz, Annals Phys. 305 (2003) 96 [arXiv:hep-th/0210184].

[52] Y. K. Cheung, M. Laidlaw and K. Savvidy, JHEP 0412 (2004) 028 [arXiv:hep-th/0406245].

[53] V. A. Rubakov and P. G. Tinyakov, Phys. Usp. 51 (2008) 759 [arXiv:0802.4379 [hep-th]].

[54] M. V. Bebronne, arXiv:0910.4066 [gr-qc].

[55] G. Dvali, O. Pujolas and M. Redi, Phys. Rev. Lett. 101 (2008) 171303 [arXiv:0806.3762 [hep-th]]. 